\title{
A review of drug delivery systems based on nanotechnology and green chemistry: green nanomedicine
}

This article was published in the following Dove Press journal:

International Journal of Nanomedicine

I2 April 2017

Number of times this article has been viewed

\section{Hossein Jahangirian' \\ Ensieh Ghasemian \\ Lemraski $^{2}$ \\ Thomas J Webster' \\ Roshanak Rafiee- \\ Moghaddam ${ }^{3}$ \\ Yadollah Abdollahi ${ }^{4}$}

'Department of Chemical Engineering, Northeastern University, Boston, MA, USA; ${ }^{2}$ Department of Chemistry, Faculty of Science, llam University, Ilam, Iran; ${ }^{3}$ School of Chemical Sciences and Food Technology, Faculty of Science and Technology, Universiti Kebangsaan Malaysia, Selangor, ${ }^{4}$ Department of Electrical Engineering, Faculty of Engineering, University of Malaysia, Kuala Lumpur, Malaysia
Correspondence: Hossein Jahangirian Department of Chemical Engineering, 313 Snell Engineering Center, Northeastern University, 360 Huntington Avenue,

Boston, MA 021I5, USA

Tel +l 982144632143

Email kamran.jahangirian@gmail.com
Abstract: This review discusses the impact of green and environmentally safe chemistry on the field of nanotechnology-driven drug delivery in a new field termed "green nanomedicine". Studies have shown that among many examples of green nanotechnology-driven drug delivery systems, those receiving the greatest amount of attention include nanometal particles, polymers, and biological materials. Furthermore, green nanodrug delivery systems based on environmentally safe chemical reactions or using natural biomaterials (such as plant extracts and microorganisms) are now producing innovative materials revolutionizing the field. In this review, the use of green chemistry design, synthesis, and application principles and eco-friendly synthesis techniques with low side effects are discussed. The review ends with a description of key future efforts that must ensue for this field to continue to grow.

Keywords: green chemistry, cancer, drug delivery, nanoparticle

\section{Introduction}

Improving human health is currently undergoing an explosion of attention led by the use of nanoparticles (NPs) to deliver drugs to cells. Such NPs are engineered so that they are attracted specifically to diseased cells, which allows for the direct treatment of those cells, improving efficacy, decreasing side effects, and overall improving human health. This technique reduces the side effects of drugs in the body. However, despite the promise of nanomedicine across all diseases, there are a number of disadvantages for using these nanodrug delivery vehicles, which should not be ignored. Drugs delivered from nanoscale entities may behave differently from when delivered in normal or conventional form. As an example, the capture of intravenously injected poly(D,L-lactide-co-glycolide) (PLGA) NPs for drug delivery in rats was shown to be considerably decreased by liver Kupffer cells as compared to the free drug. ${ }^{1}$ Coating magnetic NPs with natural polymers (such as carbohydrates and proteins) is also common. In addition, many natural polymers are biocompatible and are therefore suitable for coating NPs for biomedical applications such as cancer treatment. ${ }^{2,3}$

Despite the widespread development of nanotechnology and nanomaterials throughout the last $10-20$ years, only recently has their potential toxicological effect on humans, animals, and the environment received some attention. Moreover, although the original intended use of nanomedicine was to improve human health, NPs can be purposely misused for other intentions as many researchers have been reporting due to soiling or maximizing the toxicity of the NPs. ${ }^{4,5}$ Too often, as well, NP or nanomaterial (such as carbon nanotubes [CNTs]) synthesis techniques involve 
toxic materials. In order to decrease the cost of nanodrug delivery vehicles, make them more effective in the body, promote a healthy environment, and reduce unintended use, new approaches and design principles are clearly needed for this field.

One of the newest and most innovative approaches for manufacturing improved nanopharmaceuticals that can combat the problems noted earlier relies on green technologies. Green chemistry includes all branches of chemistry but with a strong focus on the synthesis of chemical compounds and chemical engineering processes in industrial applications based on natural materials. In contrast, the main rules of green chemistry also affect laboratory experiments, preparing for a safer environment. Based on green chemistry, or sustainable chemistry, the application and generation of hazardous materials are minimized during reaction and synthesis. Green chemistry also includes methods to produce renewable substances. The main goals of green chemistry are as follows: the design of reactions of the greatest efficiency, the use of renewable materials and energy sources, the use of safe solvents or reactants, and the prevention of waste production. Green nanotechnology has referred to the application of the 12 principles of green chemistry to design new nanomaterials to achieve economic, social, health, and environmental benefits. In this review, we considered the use of green chemistry principles for the improved synthesis and modification of nanodrug delivery systems. Among the large number of articles that discuss the synthesis and application of nanodrug delivery systems, we can find a few articles that have tried to consider one or more of these green chemistry principles. Such studies by these researchers help to draw more attention to the worthy objectives of green chemistry, as we hope this review will as well.

In this review, we consider NP research that has covered the abovementioned goals, coining a new term "green nanomedicine". The review begins with a description of the green synthesis of several nanodrug delivery vehicles. The vast majority of examples for the application of green chemistry for nanodrug carriers rely on gold NPs (AuNPs), polymer NPs, and biological drug delivery vehicles based on proteins and lipids; this will be emphasized first. A brief description on the green manufacturing procedures under development will be given following every section. Finally, an emphasis will be placed on the future directions that the field of green nanochemistry needs to take for the eventual widespread use of green chemistry in nanomedicine to treat a wide range of diseases safely and effectively.

\section{Production of NPs by green chemical reactions \\ Nanometal compounds}

In recent years, green chemistry has been introduced for the synthesis of metal nanodrugs in many fields. Traditional processes for the synthesis of metal NPs sometimes produce large quantities of toxic as well as unnecessary and harmful substances. ${ }^{1}$ However, such metallic NPs have shown great promise in medicine. For example, maghemite NPs $\left(\gamma-\mathrm{Fe}_{2} \mathrm{O}_{3}-\mathrm{NPs}\right)$ and magnetite NPs $\left(\mathrm{Fe}_{3} \mathrm{O}_{4}\right.$-NPs $)$ have been extremely helpful in drug delivery, treatment, and imaging of integrins on tumor cells as well as therapeutic outcomes. ${ }^{2-4}$ As an example, therapeutic compounds have been attached to biocompatible nontoxic superparamagnetic and magnetic NPs and injected into the body, while magnetic fields are generated outside of the body to focus their accumulation on specific targets in vivo. Iron oxide NPs have attracted great interest due to their super magnetic properties, high surface area, biocompatibility, and healthy and easy degradability. The use of iron oxide magnetic NPs for inductive hyperthermia, chemotherapy, gene carriers for gene therapy, magnetic sensing probes for in vitro diagnostics, vaccines, antibody agents, and therapeutic agents for hyperthermiabased cancer treatments has already been studied.

However, through such studies, particularly in vivo, the field has learned of some of the harmful consequences of using magnetic NPs. For example, the efficient treatment of brain disorders needs careful consideration due to drugs and NPs crossing the blood-brain barrier (BBB). The medicinal properties of iron oxide NP anions and the sufficiency of lysophosphatidic acid (LPA) to temporarily disrupt tight junctions and allow iron oxide NP anions to enter brain cells have created excitement for the treatment of neural diseases as well as caution for the unintended iron accumulation in the brain. Under normal conditions, iron oxide NP anions have a plasma half-life of 6 minutes, with the liver and spleen being the major organs of deposition. Treatment with LPA modified with iron oxide NP anions in the brain and spleen in mice revealed no signs of peripheral immune cell infiltration in the brain and no significant activation of microglia or astrocytes. The study showed improved delivery efficiency of iron oxide NP anions following LPA administration. Such findings suggest a temporary disruption of the $\mathrm{BBB}$, which may be safe and effective for increasing iron oxide NP anion delivery to the brain. ${ }^{5}$

Recently, magnesium (Mg) NPs have been shown to have extraordinary merit in terms of modifying therapy exclusivity 
and thermal ablation effects on tumors. Furthermore, the low toxicity and side effects of Mg-based chemistries that remain in the body after surgery are noteworthy. ${ }^{6}$ Some of the main advantages of magnetic nanocompounds for hyperthermiabased therapy and controlled drug delivery are given in Figure 1.

Biocompatible and monodispersed iron oxide superparamagnetic NPs were modified using folic acid (FA), which was chosen as the targeting agent combined with poly(ethylene glycol) (PEG) by Zhang et al. The biological study showed that modifying NPs with PEG-FA significantly improved the intracellular uptake of NPs by target cells. ${ }^{7}$ Drug-delivery vehicles based on two ring-type soft magnets, and a simple plastic hinge is another example for active locomotive intestinal capsules. The present drug delivery vehicle has good drug-loading capability and can conquer unfavorable distribution throughout the body, which is common for other drug delivery vehicles of much larger size. ${ }^{8}$

Sometimes, the modification of NP properties is used to decrease harmful effects. For example, biodegradable iron stents have great potential to reduce limitations, such as chronic inflammation and premature recoil. White et al studied the safety and efficacy of biodegradable iron and cobalt chromium stents in porcine coronary arteries randomly placed in the coronary arteries of juvenile domestic pigs.
Their results did not show any impressive differences between iron and cobalt chromium stents. These biocorrodible iron stents are some of the most important examples of a safe nanomaterial, helping to decrease side effects. ${ }^{2}$

The development of biodegradable drug delivery compounds with good targeted recognition and controlled release has led to the fabrication of polyphenol-coated porous nanomaterials (eg, silica NPs) without using any other organic target for cancer therapy. The supported polyphenol with colloidal stability and low premature drug leakage produced a scaffold for the immobilization of targeting moieties, such as aptamers. ${ }^{9}$ To effectively deliver antitumor drugs to the cancer cells and reduce its toxic side effects on normal cells, a PEG shielding and tumor microenvironment triggering a cascade of a pH-sensitive hollow mesoporous silica NP (HMSN) drug delivery system was prepared. 3-(3,4-Dihydroxyphenyl) propionic acid-functionalized beta-cyclodextrin $(\beta-C D)$ was loaded onto the surfaces of HMSNs using boronic acid-catechol ester bonds. Then, PEG-conjugated adamantine (Ada) was harbored onto a HMSNs- $\beta-C D$ nanocarrier via the host-guest interaction. In vivo results confirmed that the drug-loaded HMSNs prevented tumor growth considerably with minimal toxic side effects. This method introduced new insights into the extension of the new production of green chemistry-derived drug delivery carriers by the tumor microenvironment. ${ }^{10}$

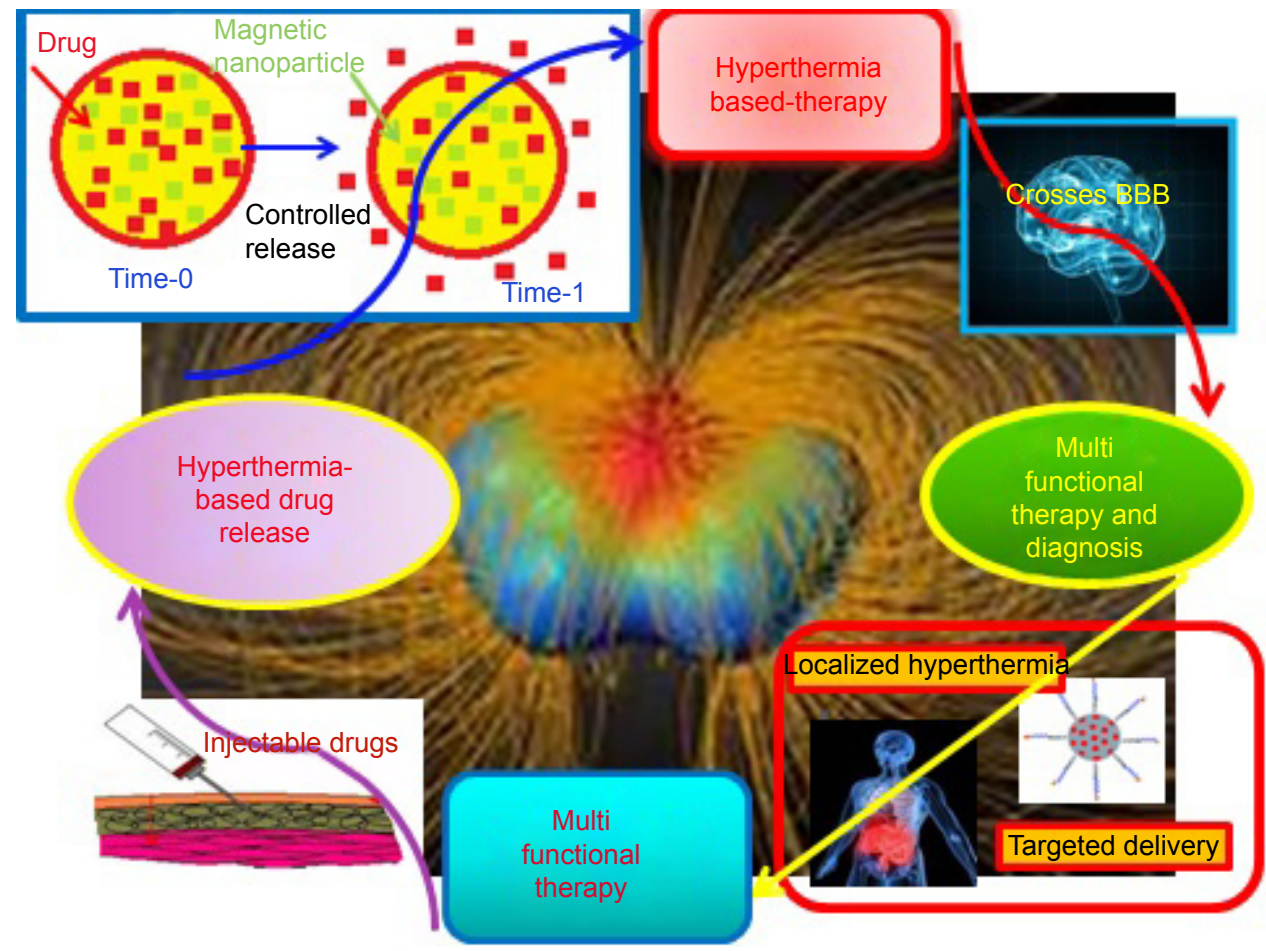

Figure I A schematic representation of some of the unique advantages of magnetic nanomaterials for hyperthermia-based therapy and controlled drug delivery. Abbreviation: BBB, blood-brain barrier. 
Other favorable green polymers have been prepared based on polyacrylate/nanosilica. However, polyacrylate coatings have promoted pleural effusion, pericardial effusion, and pulmonary fibrosis and granuloma, which are thought to be due to the high disposal of the NPs in the loading process. ${ }^{11}$

NPs have increasingly been used for numerous industrial purposes. However, concerns about their toxic and harmful effects on humans and the environment have been raised. Some research has determined the presence of NPs in patient biopsies and has reported the potential injurious effects to human lungs. Using electron microscopy and energydispersive X-ray analysis, as an example, silica NPs were identified in macrophages, pulmonary microvessels, vascular endothelial cells, microlymphatic vessels, and pleural effusions and a few in alveolar epithelial cells and pulmonary interstitial tissues (with no microscale particles present) and have been shown to damage alveolar epithelial cells, macrophages, vascular endothelial cells, and the blood-gas barriers. Based on the well-documented toxicity of the silica nanocompound, it is possible that these silica NPs may be related to some of the illness reported by researchers. ${ }^{12}$

Some nanopolymer matrices prepared by the chemical reduction of metal ions in an aqueous medium have been used as drug carriers. These novel hydrophilic healthy nanocomposites containing silver NPs (AgNPs) have been composed of a functional polymer matrix of poly(1-vinyl-1,2,4-triazoleco- $N$-vinylpyrrolidone) (poly[VT-co-VP]) prepared by free radical-initiated polymerization. Due to their sufficient stabilization by functional groups, AgNPs do not precipitate and/or change in size even after storage in aqueous medium. The toxicity of the initial poly(VT-co-VP) copolymer and nanocomposite containing AgNPs was identified on white mice, its value being $>5,000 \mathrm{mg} / \mathrm{kg}$. The nanocomposite had a pronounced antimicrobial activity toward different strains of Gram-negative and -positive bacteria. The minimum inhibitory concentration (MIC) decreased the growth of the microorganisms at concentrations ranging from 0.5 to $8 \mu \mathrm{g} / \mathrm{mL}$, and the minimum bactericidal concentration (MBC) ranged from 0.5 to $16 \mu \mathrm{g} / \mathrm{mL}$. So, these new silver (Ag) nanocomposites in a poly(VT-co-VP) matrix are promising carriers for hydrophilic antiseptics and antimicrobial drugs for medical applications to treat many infectious diseases, including surgically acquired ones. ${ }^{13}$

A molybdenum disulfide $\left(\mathrm{MoS}_{2}\right)$ nanosheet was developed as a photothermal agent for tumor therapy. Surface modification of PEG has been accomplished during a facile approach to prepare soybean phospholipid-encapsulated $\mathrm{MoS}_{2}\left(\mathrm{SP}-\mathrm{MoS}_{2}\right)$ nanosheets with excellent colloidal stability. By not observing clear in vitro and in vivo hemolysis, coagulation, and cyto/ histo-toxicity, the SP-MoS 2 nanosheets illustrate good photothermal conversion performance and photothermal stability during breast tumor photothermal therapy. The prepared SP-MoS ${ }_{2}$ nanosheets showed low cost, simple fabrication, and good in vivo hemo/histo-compatibility, supporting a promising capacity for the treatment of cancer. ${ }^{14}$ Figure 2 shows a schematic illustration of the synthesis of SP-MoS .

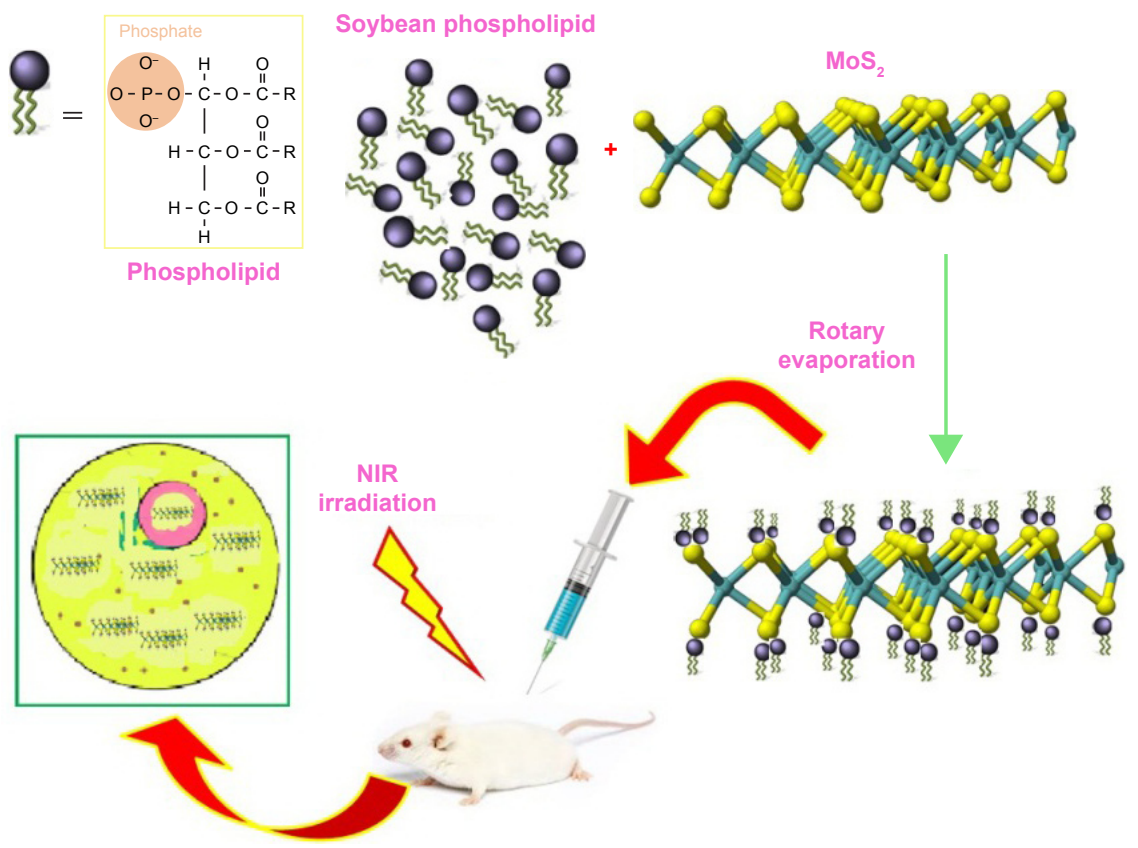

Figure 2 Schematic illustration of the synthesis of soybean phospholipid-encapsulated $\mathrm{MoS}_{2}$. Abbreviations: $\mathrm{MoS}_{2}$, molybdenum disulfide; NIR, near infrared absorbing dyes. 
Sometimes, bovine serum albumin (BSA) has been used to modify NaGdY4-based upconversion NPs (UCNPs). The obtained UCNP@BSA NPs showed excellent solubility in water as well as physiological solutions. Utilizing the hydrophobic domains in the BSA protein, two different dye molecules, including a photosensitizer, Rose Bengal (RB), and near infrared absorbing dyes, IR825, can be effectively loaded onto UCNP@BSA NPs. On one hand, the obtained dual-dye-loaded NPs could serve as a dual modal magnetic resonance (MR) and upconversion optical imaging probe while, on the other hand, are effective in both photodynamic and photothermal therapies, which if combined together, could result in excellent synergetic cancer-killing properties both in vitro and in vivo. ${ }^{15}$

A summary of the applications of green chemistry principles for nanometal drug delivery is given in Table 1.

\section{Polymer nanocomposite (PNC)}

A PNC containing a polymer or copolymer with NPs dispersed in the polymer matrix is one way to decrease toxicity and increase safety. These nanocomposites of course can have different shapes (eg, platelets, fibers, and spheroids) but should have at least one dimension in the range of nanometers. Polymeric NPs have been prepared for decades for use in a variety of high-performance materials such as nanodrug delivery or functionalized with drugs, showing their safety if the proper chemistry is used.

To introduce a novel and healthy drug candidate for treating endometriosis, a new polymeric nanogene delivery vehicle consisting of polyethylenimine-grafted chitosan oligosaccharide (CSO-PEI) with hyaluronic acid (HA) and small interfering RNA (siRNA) was prepared. There was no clear difference in size observed between (CSO-PEI/siRNA) $\mathrm{HA}$ and CSO-PEI/siRNA, but the fluorescence accumulation in the endometriotic lesion was more important for (CSO$\mathrm{PEI} / \mathrm{siRNA}$ )HA than for CSO-PEI/siRNA due to the specific binding of HA to CD44. In addition, the (CSO-PEI/siRNA) HA NP gene therapy significantly reduced the endometriotic lesion size with atrophy and degeneration of the ectopic endometrium. The epithelial cells of the ectopic endometrium from rat models of endometriosis showed a significantly lower CD44 expression than controls after treatment with (CSO-PEI/siRNA)HA. Furthermore, observations under an electron microscope showed no obvious toxic effects on reproductive organs and confirmed that the (CSO-PEI/ siRNA)HA gene delivery system can be used as a safe and effective way for the treatment of endometriosis. ${ }^{16}$ Additional research related to molybdenum NPs includes phosphate glasses incorporating vanadium and molybdenum oxides as useful compounds for the control of drug dissolution and drug release. The modified vancomycin molecules demonstrate greater hydrogen bonding with vanadium-doped glasses and consequently slower drug release over 14 days showing an improved surface modification with drug molecules. This can be described by the strong coherence of drug components to the glass surface compared to the free molybdenum. The strong attachment is due to hydrogen bonding between the amino functional groups of vancomycin and the hydrated $\mathrm{P}-\mathrm{O}-\mathrm{H}$ groups in the glass network. ${ }^{17}$

A prepared nanogel from dextran and poly(lactide) by in situ cross-linking with a homobifunctional cross-linker through a conventional radical polymerization technique is another compound to increase NP efficiency. Degradation experiments using hen egg lysozyme (Ly) confirmed the biodegradability of the nanogel. In vitro cytocompatibility studies against human mesenchymal stem cells (hMSCs) proposed that the native nanogel had nontoxic effects on cancer cells, while doxorubicin (DOX)-loaded nanogels demonstrated high toxicity toward cancer cells. ${ }^{18}$ The aforementioned nanogel, injectable, multifunctional, biodegradable PLGA was synthesized and tested on breast cancer cells. These particles were loaded with an anticancer drug from prodigiosin (PG), which was obtained from bacteria, Serratia marcescens subsp. and Serratia marcescens. ${ }^{19}$ A novel reactive oxygen species-cleavable diblock polymer was prepared with hydrophilic PEG shells and a hydrophobic PLGA core. This new nanocompound with high stability, efficient drug delivery, excellent sensitivity, and good compatibility has been used for

Table I A summary of the application of green chemistry principles for nanometal drug delivery

\begin{tabular}{|c|c|c|c|}
\hline Nanometal drug delivery vehicles & Use & Green chemistry principle & References \\
\hline Biocorrodible iron stents & Porcine coronary arteries & Designing safer chemicals & 2 \\
\hline Basal fibroblast growth factor-iron oxide nanoparticles & Cancer radiation therapy & Designing safer chemicals & 5 \\
\hline Magnesium nanoparticles & Hyperthermia therapy & Design for degradation (biodegradable) & 6 \\
\hline Iron oxide coating with PEG & Targeting agent & Atom economy & 7 \\
\hline Hollow mesoporous silica nanoparticle- $\beta-C D$ nanocarrier & Inhibited tumor growth & Less hazardous chemical syntheses & 10 \\
\hline AgNPs in a polymer & Antiseptics and antimicrobial & Safer solvents and auxiliaries & 13 \\
\hline Molybdenum disulfide nanosheet & Photothermal agent & Less hazardous chemical synthesis & 14 \\
\hline
\end{tabular}

Abbreviations: AgNPs, silver NPs; $\beta-C D$, beta-cyclodextrin; NPs, nanoparticles; PEG, poly(ethylene glycol). 
cancer chemotherapy. ${ }^{20}$ Different size ranges of poly(tannic acid) particles, prepared by using lecithin/gasoline microemulsion media, showed high antimicrobial activity against a wide spectrum of bacteria and yeast strains, such as Staphylococcus aureus, Escherichia coli, Pseudomonas aeruginosa, and Candida albicans. ${ }^{21}$ Based on green chemistry principles, the use of safe materials (eg, solvents and separation agents) reduces the waste from chemical reactions. The hydrophilichydrophobic character of the polyester dendron-polymer leads to the formation of flower-like micelles in aqueous solution. All nanocopolymer compounds were effective for passive tumor targeting through the enhanced permeability and conservation effect. ${ }^{22}$ Cardiovascular diseases, including ischemic heart disease and stroke, are responsible for $\sim 25 \%$ of all deaths worldwide. Easy availability of nanobiomaterials increases their application as drug delivery vehicles or protective nanoshells improving the biocompatibility of imaging agents. Poly-L-lactic acid (PLLA) stents are better than metallic stents due to their biodegradability and local delivery of drugs. Moreover, the feasibility and safety of coronary biodegradable polymer stents composed of PLLA in humans have been demonstrated. Results have not shown any major clinical events related to PLLA stent implantation in up to 15 patients after 1 month of implantation. After 6 months, all patients showed acceptable restenosis and target lesion revascularization rates without deaths, myocardial infarctions, or coronary artery bypass grafting recorded. ${ }^{23}$

PEG-modified thiolated gelatin (PEG-SHGel) NPs were developed as a long-circulating passively targeted delivery system that responds to intracellular glutathione (GSH) concentrations to enhance DNA delivery and transfection. Reporter plasmid expressing enhanced green fluorescent protein (EGFP-N1) was encapsulated in the NPs. The PEGSHGel NPs released encapsulated plasmid DNA in response to varying concentrations of GSH. The results of this study showed that PEG-SHGel NPs could serve as a very efficient nanoparticulate vector for systemic DNA delivery to solid tumors where the cells are known to have significantly higher intracellular GSH concentrations. ${ }^{24}$

New drug-eluting stents (DES) have been developed to overcome the relatively high rates of restenosis and target lesion revascularization with bare metal stents. A new and absorbable-coating sirolimus-eluting stent (AC-SES) was prepared for its ability to carry drugs more efficiently within the intimal area rather than concentrating the drug around the stent struts and for its capacity to match supporting erosion with drug release. The supporting struts consisted of absorbable PLGA and crystalline sirolimus deposited by a dry-powder electrostatic process. The AC-SES results showed acceptable drug stability under simulated conditions and consistent drug delivery in a porcine coronary implant model. ${ }^{25}$ There have also been a significant number of research efforts to identify whether corrodible substances may be applied as low toxic biodegradable cardiovascular implants. Corrodible iron stents were prepared from pure iron and laser cut with a stent design similar to a commercially available used stent. Stents were implanted into the native descending aorta of New Zealand white rabbits. No thromboembolic complications and no considerable neointimal proliferation with any significant inflammatory effect or systemic toxicity occurred during a 6-18-month follow-up. So, this important in vivo study pointed that degradable iron stents can be safely implanted without important obstruction of the stented vessel caused by inflammation, neointimal proliferation, or thrombotic events. ${ }^{26}$ In this field, the toxicity of a corrodible stent prepared from pure iron in a peripheral stent was determined. The stent was implanted into the descending aorta of minipigs with an overstretch injury without technical problems. Histopathological examination of heart, lung, spleen, liver, kidney, and paraaortic lymphatic nodes confirmed no signs of iron overloaded and local toxicity of iron due to corrosion products. ${ }^{27}$ Healthy and nontoxic $\mathrm{Mg}$ stents are one of the safest ways to deliver drugs, which have been tested in extensive clinical trials on patients with acute coronary syndrome, myocardial infarction, and diabetes without any side effects. ${ }^{28,29}$

A new biodegradable and safe copolymer for NP incorporation was prepared using a reaction of polycondensation and ring-opening polymerization of $\varepsilon$-caprolactone in the presence of poly(propylene adipate). The prepared nanocomposite showed low cytotoxicity and was used to deliver desferrioxamine, an iron-chelating drug. ${ }^{30}$

In addition, a novel and very soluble electrosprayed nanospherule was prepared based on poorly water-soluble fenofibrate. Numerous fenofibrate-loaded electrosprayed nanospherules were synthesized with polyvinylpyrrolidone (PVP) and applied in drug delivery using the electrospray technique. Results confirmed that the electrosprayed nanospherule method remarkably increased aqueous solubility and dissolution compared to the free drug. ${ }^{31}$ Similar results have been reported by Withey's group for the synthesis of core-shell micelle delivery vehicles for cobalt-based pharmaceuticals. The cobalt complex was synthesized during the self-assembly of a poly(trimethylsilyl propargyl methacrylate)- $b$-poly(PEG methyl ether methacrylate). ${ }^{32}$ Recently, biodegradable poly(ester amine) (PEA)- and poly(amido amine) (PAA)-based nanocomposites were 
prepared for efficient siRNA delivery to human umbilical vein endothelial cells (HUVECs). The effects of the nanocomposites on small molecule delivery to cells were determined and compared with research using DNA-containing particles. Results demonstrated that PEAs and linear nondendrimeric PAAs were very effective for DNA delivery to HUVECs using green fluorescent protein-encoding plasmid DNA (up to $50 \%-60 \%$ transfection efficiency). ${ }^{33}$

However, the deficiencies of the delivery of small antitumor molecular drugs have been significantly improved by entrapping such small molecules into nanovehicles as well as controlling drug release and tumor responsiveness of the drug nanocarriers. The use of the core cross-linking method to synthesize a micelle with enhanced drug encapsulation ability and sensitivity of drug release in tumors has been shown. For example, a micelle composed of PEG and FA-PEG as the hydrophilic units, pyridyldisulfide as the cross-linkable and hydrophobic units, and the disulfide bond as the cross-linker has shown considerable promise for cellular uptake, in vitro smart tumor responsiveness, and cytotoxicity of core cross-linked micelle-curcumin against HeLa cells improved. ${ }^{34}$ Various types of drug-delivering vehicles between noncross-linked micelle (NCM) and folic acid-core cross-linked micelles are shown in Figure 3. Another synthesized nanodrug delivery vehicle based on green chemistry is $\mathrm{pH}$-sensitive DOX encapsulated in the hydrophobic cores of the amphiphilic triblock copolymers. The copolymer micelles entered into lysosomes using a lysosome tracker method. These safe and effective micelles can be used as a hopeful treatment for enhanced intracellular drug delivery and real-time imaging. ${ }^{35}$

A new, highly water-soluble poly(L- $\gamma$-glutamylglutamine)-paclitaxel (PGG-PTX) nanoconjugate has been modified to improve the therapeutic index of paclitaxel (PTX). PGG-PTX is an enhancement of the poly(L-glutamic acid)-PTX (PGA-PTX) conjugate in which additional glutamic acid is linked to each glutamic side chain in the polymer. This process increases water-solubility of PGAPTX. Unlike PGA-PTX, during self-assembly into NPs, the size of the PGG-PTX nanocomposite does not change over suitable concentrations. The potential of PGG-PTX during treatment of a human lung cancer H460 cell line was acceptable to other known polymer-PTX conjugates. Result confirmed that the maximum sustained dose of PGG-PTX was higher than the maximum sustained dose of the PGA-PTX and PGG-PTX was substantially higher than PGA-PTX. ${ }^{36}$ The study concerning the therapeutic effect of a synthesized tricalcium phosphate (TCP) nanocomposite material (nanoTCP) on hepatocarcinoma in a rat model confirmed the safety and biodegradability of this nanocomposite. Results from the histopathological examination of liver tissues were compatible with the biochemical observation and confirmed

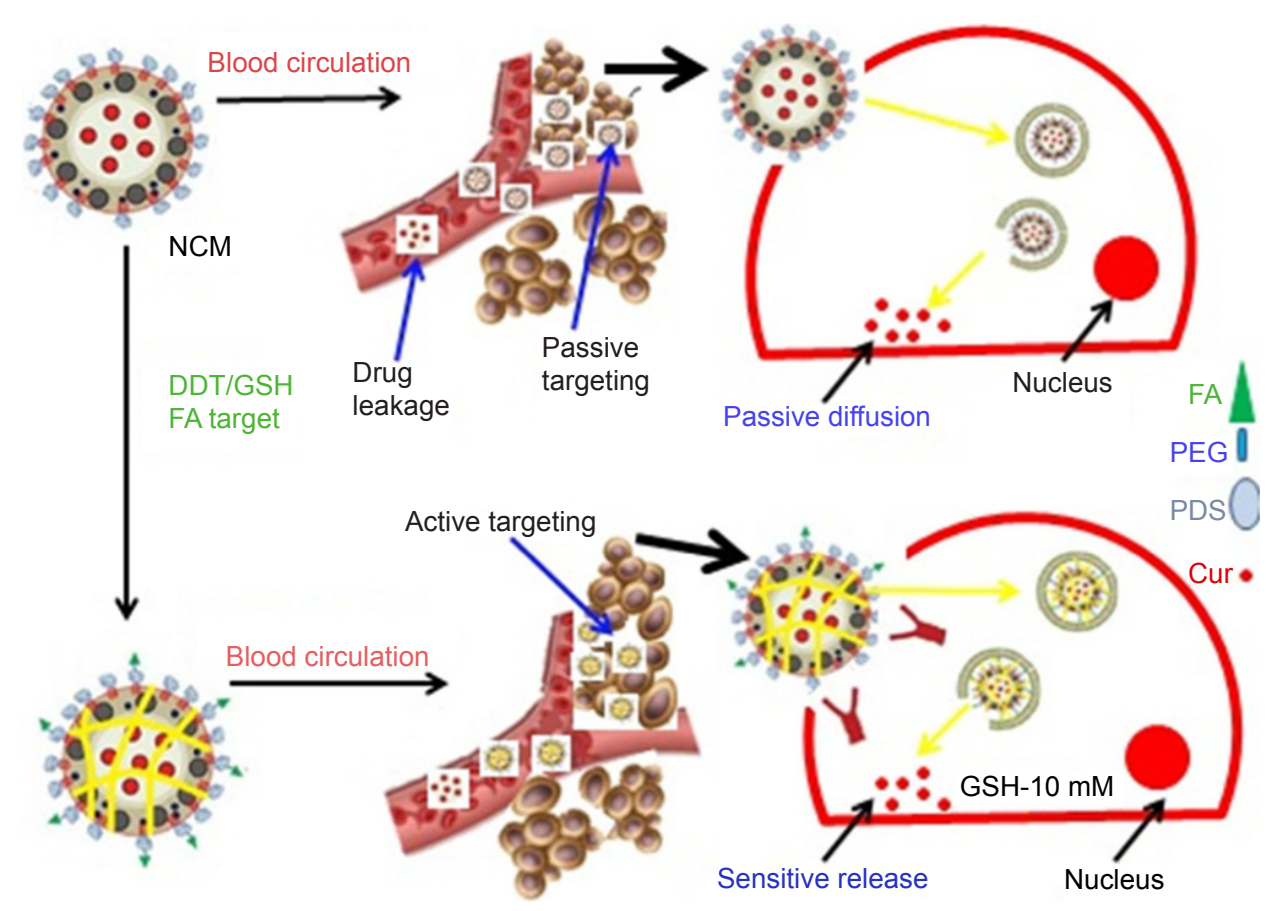

Figure 3 Schematic image of different drug-delivering vehicles and releasing techniques between NCM and FA-CCM. Abbreviations: FA, folic acid; GSH, glutathione; PEG, poly(ethylene glycol); Cur, curmine; NCM, noncross-linked micelle; PDS, pyridyldisulfide; DTT, dithiothreitol. 
the difference between the control and treated groups. In total, it was found that nano-TCP is a good candidate for curing hepatocarcinoma completely and presenting a new choice for patients to receive healthy, fast, and effective treatment. ${ }^{37}$

Another example of a green medical compound is NP formulations with chitosan. Chitosan-graphene oxide (GO) green nanocomposite films were prepared by mixing aqueous chitosan solutions and GO in dilute acetic acid as a solvent. The nanocomposite included quaternized chitosan (QC)/BSA/rectories (REC-DOX was used to investigate the entrapment efficiency and release pattern in NPs). Results showed that using REC could increase drug encapsulation ability and loading potential. ${ }^{38}$ Pancreatic cancer is a lethal malignancy the progression of which is highly dependent on nervous system microenvironments. Recently, neural drugsupported ferritin NPs (Ft NPs), which regulate the nervous microenvironment, have also been shown to control pancreatic cancer progression. The drug-supported Ft NPs can target pancreatic tumors via passive targeting of enhanced permeability and retention effects in tumors and active targeting via transferrin receptor 1 (TfR1) binding on tumor cells, with a triggered drug release in an acidic tumor media. Two drugs, one which activates neural activity (carbachol) and the other which impairs neural activity (atropine), were inserted into the Ft NPs to form two types of nanodrugs, Nano-Cab NPs and Nano-Ato NPs, respectively. The activation of the nervous micromedia by Nano-Cab NPs significantly prevented pancreatic tumor progression, whereas the blockage of the neural niche by Nano-Ato NPs generally destroys neurogenesis in tumors and the development of pancreatic cancer. The Ft-based NPs, thus, include a successful and healthy way of carrying neural drugs for new anticancer therapies. ${ }^{39}$

Alginate also represents a green nanomedicine approach to decrease NP toxicity. For example, quinapyramine sulfate (QS)-supported sodium alginate NPs have been prepared to decrease unpleasant toxic effects of QS against the parasite Trypanosoma evansi, a causative factor of trypanosomosis. To identify the toxicity of the novel NPs, the biocompatibility of QS-NPs was tested using Vero, HeLa cell lines, and horse erythrocytes in a dose-dependent manner and displayed no toxicity at effective trypanocidal doses and even at doses several times higher than the effective dose. ${ }^{40}$ Another example of safe, biocompatible, and bioresorbable bioactive drug delivery vehicles for the heart include calcium phosphate NPs (CaP-NPs). This new nanodrug delivery vehicle was efficiently internalized into cardiomyocytes without significant toxicity or interfering with any functional properties. ${ }^{41}$
Two-dimensional silicon NPs (Si-NPs) are another kind of nanocompound with high capacity for applications in drug delivery, biodetection, and diagnosis. Compared to amorphous colloidal and porous silica, modified or functionalized mesoporous silica showed high biocompatibility, safety, and controlled drug release. ${ }^{42}$ Biocompatible Si-NPs with amine functional groups synthesized using a one-step green protocol opens the route for further conjugation with biomolecules, which is an interesting parameter for applications of NPs in the biomedical area, such as biolabels, contrast agents, and vector delivery, and may appear as a bionanostructure for good translation into the clinical setting. ${ }^{43}$

Injectable polymethylmethacrylate (PMMA) bone cement is a widely used bone substitute in cemented arthroplasty, vertebroplasty, and osteoporosis fractures. However, due to inappropriate stiffness, poor bioactivity, and high polymerization temperature of PMMA, aseptic loosening of the implanted cement at the bone-cement interface still could be observed in a high rate of patients. To improve the performance of PMMA, artificial extracellular matrices, such as a chitosanglycerophosphate (CS-GP) thermosensitive hydrogel, were introduced into PMMA acting as a pore-forming agent and an osteoconductive nanosized hydroxyapatite (nano-HA)/ antibiotic gentamicin (GM) acting as a carrier. Results showed that the CS-GP thermosensitive hydrogel can effectively create open pores at the surface of the PMMA cement, which is believed to facilitate bone tissue ingrowth and improve the cement anchorage at the bone-cement interface in future clinical applications. More importantly, nano-HA and GM enriched the CS-GP thermosensitive hydrogel effectively to improve the overall performance of the PMMA cement without influencing cell survival, suggesting that the injectable $p$-PMMA/CS-GP/nano-HA/GM cement will hold strong promise for future bone reconstruction applications. ${ }^{44}$

Ly and sodium carboxymethyl cellulose (CMC) were used to fabricate nanogels by a convenient method without using any chemical treatment except simple heating to achieve the denaturation temperature of Ly and were used as model drugs to investigate the entrapment efficiency and release ability in nanogels. Besides, the in vitro release experiments of 5-Fu in simulated gastric (SGF) and intestinal fluids (SIF) showed that the nanogels obviously have a sustained release effect, and the release in SGF was more slow. In addition, the established method for fabricating drug delivery may be extended to tailor the size and composition of other protein and polysaccharide-based NPs with tunable release speed for various applications, such as catalysis and enzyme immobilization. ${ }^{45}$ 
Table 2 Applications of green nanocomposites in drug delivery

\begin{tabular}{|c|c|c|c|}
\hline Nanocomposite drug delivery & Use & Green chemistry principles & References \\
\hline $\begin{array}{l}\text { Polyethylenimine-grafted chitosan } \\
\text { oligosaccharide-hyaluronic acid-RNA }\end{array}$ & Gene delivery system & Designing safer chemicals & 16 \\
\hline $\begin{array}{l}\text { Phosphate glasses incorporating vanadium } \\
\text { and molybdenum oxides }\end{array}$ & $\begin{array}{l}\text { Management of dissolution and drug } \\
\text { release }\end{array}$ & Safer solvents and auxiliaries & 17 \\
\hline Dextrin-poly(lactide) nanogel & Human mesenchymal stem cells & Design for degradation & 18 \\
\hline PLGA & Breast cancer cells & Design for degradation & 19 \\
\hline ROS-cleavable diblock copolymer (MeO-PEG-TK-PLGA) & Load the anticancer drug doxorubicin & Designing safer chemicals & 20 \\
\hline Polyester dendrons & Passive tumor targeting & Safer solvents and auxiliaries & 22 \\
\hline PLLA stents & Deliver drugs & Designing safer chemicals & 23 \\
\hline PLGA-crystalline sirolimus & Deliver drugs & Less hazardous chemical syntheses & 25 \\
\hline Mg stents & Deliver drugs & Designing safer chemicals & 28,29 \\
\hline Core-shell micelle carriers & Delivery of cobalt pharmaceuticals & Designing safer chemicals & 30 \\
\hline $\begin{array}{l}\text { Poly(ester amine)- and poly(amido amine)- } \\
\text { based nanoparticles }\end{array}$ & siRNA delivery & Use of renewable feedstocks & 32 \\
\hline Folic acid-targeted disulfide-based cross-linking micelle & Deliver drugs & Safer solvents and auxiliaries & 34 \\
\hline Poly(L- $\gamma$-glutamyl-glutamine)-paclitaxel nanoconjugate & Human lung cancer & Safer solvents and auxiliaries & 36 \\
\hline Chitosan-graphene oxide composite & Deliver drugs & Safer solvents and auxiliaries & 38 \\
\hline
\end{tabular}

Abbreviations: Mg, magnesium; PLGA, poly(D,L-lactide-co-glycolide); PLLA, poly-L-lactic acid; ROS, reactive oxygen species; siRNA, small interfering RNA; MeO, methoxylpolyethylene glycol-thioketal.

A summary of the applications of green chemistry principles for PNC drug delivery systems is listed in Table 2.

\section{Green quantum dots (QDs)}

Based on special physical and chemical properties, graphene and graphene compounds have been effectively used for active and passive targeted drug delivery. A water-soluble graphene derivative, specifically GO, is currently being highly researched by researchers all around the world. For example, Gurunathan et al reported an environmentally friendly, cost-effective, and green method for the synthesis of water-soluble graphene using bacteria biomass. This reduction synthesis prevented the application of toxic reactants, such as hydrazine and hydrazine hydrate compounds, and thus, represents green nanomedicine. ${ }^{46}$

Recently, a sonochemical approach was utilized for the development of a graphene-gold $(\mathrm{G}-\mathrm{Au})$ nanocomposite. Through the sonochemical method, simultaneous exfoliation of graphite and the reduction of gold chloride occurred to produce a highly crystalline $\mathrm{G}-\mathrm{Au}$ nanocomposite. This $\mathrm{G}-\mathrm{Au}$ nanocomposite was used to modify glassy carbon electrodes (GCE) to fabricate an electrochemical sensor for the selective detection of nitric oxide (NO), a critical cancer biomarker. G-Au-modified GCE exhibited an enhanced electrocatalytic response toward the oxidation of $\mathrm{NO}$ as compared to other control electrodes. Furthermore, this enzyme-free G-Au/GCE exhibited an excellent selectivity toward NO in the presence of interferences. This G-Au nanocomposite introduced a new electrode material in the sensitive and selective detection of $\mathrm{NO}$, a prominent biomarker of cancer. ${ }^{47}$
Cancer nanotechnology encourages cutting edge research utilizing nanomaterials for the diagnosis, therapy, and prevention of cancer. Recognition of cancer-related biomarkers in the body has made early detection possible and, thus, paves the way toward devising methods to control it from progressing to advanced stages. Hydrogen peroxide $\left(\mathrm{H}_{2} \mathrm{O}_{2}\right)$ is a critical biomolecule, which plays an important dual role in cancer progression. Herein, a sensitive method for the detection of $\mathrm{H}_{2} \mathrm{O}_{2}$ utilizing a reduced $\mathrm{GO}-\mathrm{Ag}(\mathrm{RGO}-\mathrm{Ag})$ nanocomposite has been developed. This RGO-Ag nanocomposite was prepared using a green and facile one-step synthesis approach utilizing the extract of a medicinal mushroom, Ganoderma lucidum. This excellent electrochemical performance toward $\mathrm{H}_{2} \mathrm{O}_{2}$ detection could contribute to advances in current cancer diagnosis. The RGO-Ag nanocomposite was also explored as a potential antibacterial agent. Owing to its synergistic effects, $\mathrm{RGO}-\mathrm{Ag}$ showed a comparable antibacterial activity to the standard antibiotic, chloramphenicol. The combined antibacterial effects and sensing potential make this RGO-Ag nanocomposite a promising candidate for future health care. ${ }^{48}$

Another green and safe method for the synthesis of RGO was reported by using just riboflavin- 5 '-phosphate sodium salt dehydrate as a reducing reagent. The modified nano-RGO was systematically used as a nanocarrier for $\mathrm{pH}$-sensitive drug delivery. Hemolytic toxicity tests showed that the prepared nano-RGO did not possess any hemolytic activity, which confirmed its safety in numerous drug delivery processes. Doxorubicin hydrochloride (DOX $\mathrm{HCl})$, as a model drug, was successfully loaded onto the surface of 
nano-RGO via strong $\pi-\pi$ stacking interactions. Compared to conventional drug delivery vehicles, the DOX-attached nano-RGO nanohybrid showed a significant drug-carrying capacity, high stability, $\mathrm{pH}$ sensitivity, and a sustainable release of drugs. Cytotoxicity test results suggested that the prepared nanohybrid display possessed cytotoxicity to MCF-7 and A549 cells by nonspecific endocytosis mechanisms. Therefore, the reported green functionalized RGO could be a successful and safe candidate as an ideal drug delivery vehicle with controlled release when used at the correct concentration. ${ }^{49}$

Additional significant research on cadmium telluride (CdTe) QDs was recently performed by Zhu et al by preparing a compound to deliver the hypoxia-inducible factor-1 RNA into hypoxic cancer cells. The compound made of a functional shell composed of 2-deoxyglucose (DG)-PEG linked with the lipoic acid molecule, lysine, and 9-polyD-arginine by a hydrazone bond and a core of CdTe QDs. ${ }^{50} \mathrm{CdTe}$ QD-attached nanogels (QD-NGs) proved to be another type of NP with a good ability for drug delivery and cell imaging. Li et al reported the use of CdTe QDattached nanogels via a simple and green method using ultrapure water. The size of NPs changed their release and cytotoxicity. The larger particle size of QD-NGs afforded slower endocytosis than free QDs and reduced toxicity. Moreover, the polymer shell can prevent the release of free QDs and toxic $\mathrm{Cd}^{2+}$ and, thus, relieves QD toxicity. Cellular uptake studies demonstrated that the QD-NGs can preserve QDs from dissociation in the cytoplasm and sustain its native fluorescence intensity. The methotrexate (MTX)-entrapped QD-NGs can increase antitumor activity of the drug. The cytotoxicity of QD-NGs decreased compared to that of free QDs. ${ }^{51}$

The modified luminescent manganese-zinc sulfide QDs has been used as a stabilizer, in biological environments due to the existence of active binding sites. Also modified manganese-zinc sulfide QDs with chitosan have been synthesized in the aqueous solution for developing specific targeting, imaging, and drug delivering in cancer therapy. FA was incorporated as a targeting agent for the specific targeting of the manganese-zinc sulfide QDs toward cells overexpressing folate receptors. The results of cell viability and proliferation showed that the prepared nanocomposites did not have any toxicity against human breast cells. The result exhibited that the modification of the chitosanencapsulated QDs with FA increased the incorporation and binding ability of the nanodrug delivery vehicle toward folate receptor-overexpressed cells. Therefore, due to the low toxicity of the nanocomposite, manganese-zinc sulfide QDs can be applied as good candidates for simultaneous targeted drug delivery and cellular imaging. ${ }^{52}$

Carbon dots (CDs) are famous and significant nanodelivery vehicles based on their good optical and biocompatible properties. However, their practical ability in cancer therapeutics has limitations due to their insensitive surface properties in cancer cell media in vivo. A tumor extracellular microenvironment-sensitive drug delivery vehicle based on cisplatin(IV) prodrug-loaded charge-convertible CDs (CDs$\mathrm{Pt}[\mathrm{IV}]-\mathrm{PEG}-[\mathrm{PAH} / \mathrm{DMMA}]$ ) was prepared for image-guided drug delivery. In vivo experiments of charge-convertible CDs exhibited high tumor inhibition ability and low side effects as a smart nanodrug delivery with impressive therapeutic effects. ${ }^{53}$ Bright green luminescent graphitic carbon nitride QDs (GCNQDs) doped with oxygen and sulfur were synthesized using citric acid and thiourea via a simple microwave method. The cell viability assay of GCNQDs demonstrated that GCNQDs can be used as a good and enhanced fluorescent probe for HeLa cell imaging with low cytotoxicity and high potential in bioanalysis and related research. ${ }^{54}$ A green method for preparing semiconductor copper indium sulfide QDs in aqueous media with biocompatible GSH, as a capping ligand and stabilizer, has been reported. In addition, the synthesized QDs were safe, with no significant changes in cell viability. With arginine-glycine-aspartic acid bioconjugation, the $\mathrm{CuInS}_{2}$-RGD QDs have exhibited very good biocompatibility and good bioimaging performance. ${ }^{55}$ Figure 4 shows a schematic diagram for preparing hydrophilic $\mathrm{CuInS}_{2}$ QDs for in vitro bioimaging.

A summary of the application of green chemistry principles for QD drug delivery is given in Table 3.

\section{Biosynthesis of nanodrug delivery vehicles}

The current development in the biosynthesis of nanocarriers, specifically the synthesis of nanocompounds with specific sizes and shapes, is leading to the progression of novel NPs in cancer therapeutics and diagnosis (eg, drug and gene delivery, imaging, phototherapy, and radiotherapy enhancement treatments). Furthermore, some significant properties, such as high inherent safety, high surface area, and tunable stability, of NPs are very valuable in numerous medical and drug delivery applications. ${ }^{56-58}$

Using a new solvent for the assay of drug release from a polysaccharide-based nanocompound for a colon-specific drug delivery system is an eco-friendly and cost-effective method for the preparation of applicable nanomaterials using 


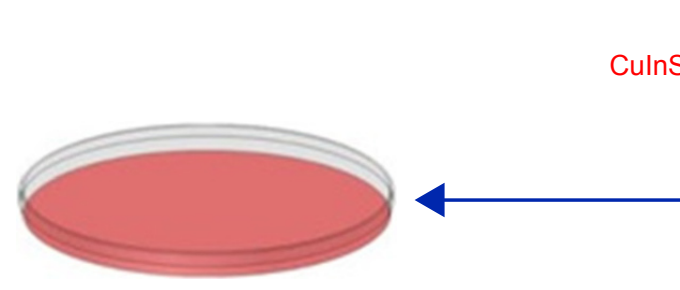

In vitro

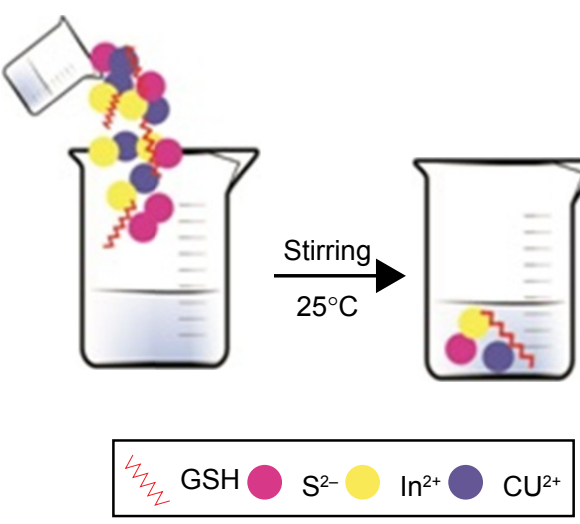

(
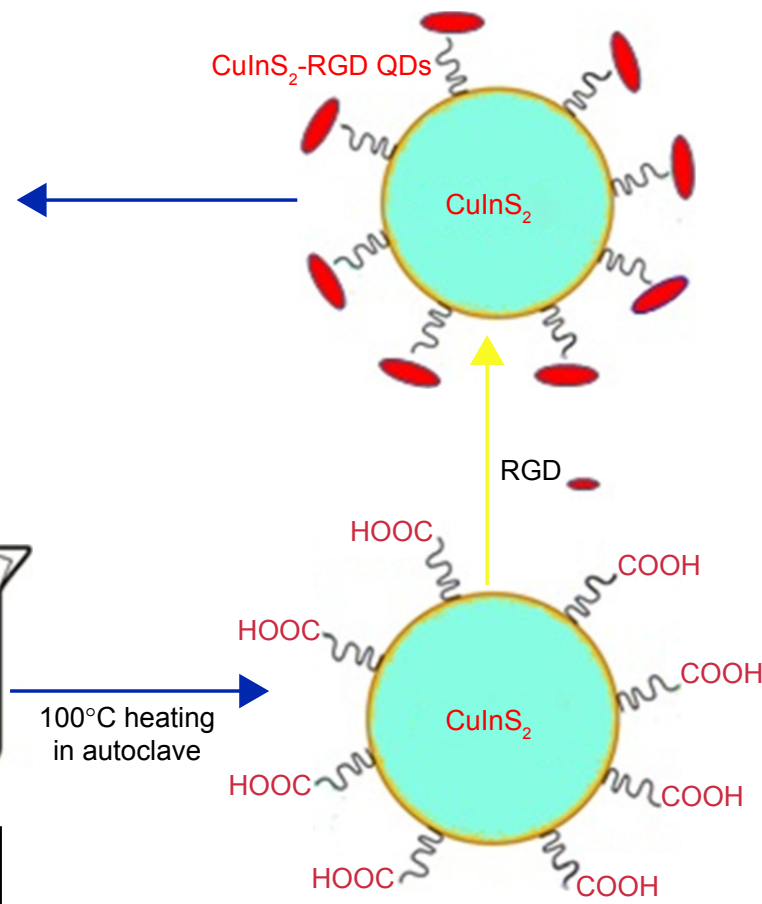

Hydrophilic QDs

Figure 4 A schematic illustration for the synthesis of hydrophilic CulnS $\mathrm{Q}_{2}$ QDs for in vitro bioimaging. Abbreviations: GSH, glutathione; QDs, quantum dots.

biomaterials. For this, probiotic cultures of Bacteroides, Bifidobacterium, Lactobacillus species, Eubacterium, and Streptococcus present in the colonic region were produced and placed in the solvent media and compared with those obtained from rat cercal- and human fecal-based fermentation model. The obtained results with the probiotic system indicated that a probiotic dissolution method can be used for the drug release of any polysaccharide-based oral formulation meant for colonic delivery. ${ }^{59}$

AuNPs and other noble metal NPs have a fascinating and progressing concern in different fields of study due to their interesting nanostructures and properties. For example, Malathi et al described the green synthesis of AuNPs for drug delivery based on chitosan as a reducing/ capping agent. They used single oil-in-water $(\mathrm{O} / \mathrm{W})$ emulsions for synthesizing a biocompatible controlled release of rifampicin in the body. ${ }^{60}$ Another safe, cost-effective, and environmentally friendly method to prepare AgNPs in aqueous media was displayed using $\mathrm{Ag}$ nitrate $\left(\mathrm{AgNO}_{3}\right)$ and freshly extracted egg whites. The results exhibited that biomolecule-loaded AgNPs have mainly spherical shapes and the average sizes of $20 \mathrm{~nm}$. The proteins of egg whites, with different functional groups, presented great tasks in reducing $\mathrm{Ag}^{+}$to produce a stable product. In vitro cytotoxicity tests showed that $\mathrm{Ag}$-protein nanocompounds exhibited acceptable biocompatibility with mouse fibroblast cells during cancer radiation therapy. ${ }^{61}$ The significant role of seaweeds as a marine origin for the biomimetic preparation of nanometal particles has increased due to the increasing needs for pharmacological products, such as antibacterial, antifungal, antiviral, and antitumor properties. The bioreduction of bulk gold chloride to prepare AuNPs in

Table 3 Applications of green chemistry principles for quantum dot drug delivery

\begin{tabular}{|c|c|c|c|}
\hline Quantum dot drug delivery & Use & Green chemistry principles & References \\
\hline Graphene oxide & $\mathrm{pH}$-sensitive drug delivery & Designing safer chemicals & 49 \\
\hline CdTe quantum dots & Deliver hypoxia-inducible factor-I RNA & Designing safer chemicals & 50 \\
\hline Manganese-zinc sulfide quantum dots & Specific targeting, imaging, and drug delivering & Less hazardous chemical syntheses & 52 \\
\hline Carbon dots & Imaging-guided drug delivery & Less hazardous chemical syntheses & 53 \\
\hline Graphitic carbon nitride quantum dots & Cell imaging & Safer solvents and auxiliaries & 54 \\
\hline
\end{tabular}

Abbreviation: CdTe, cadmium telluride. 
trivalent aurum solutions with various broth concentrations of brown seaweed (Padina gymnospora) leaf extracts has been synthesized, and AuNPs have been used in lung and liver tumor cells, without any side effects. ${ }^{62}$

Liver-specific biopolymers have been selected as a new liver cancer-targeted drug carrier with high efficacy and low side effects. Pullulan-supported AuNPs were linked with 5-fluorouracil and FA to produce new targeted drug delivery and imaging of cancer. The in vitro cytotoxicity of free Pullulan-AuNPs on tumor cells showed that the amount of Pullulan-AuNPs required to achieve $50 \%$ of growth of inhibition was much lower. ${ }^{63}$ A schematic diagram for the synthesis of Pullulan-AuNPs is shown in Figure 5.

Biodegradable polymers are an important group of macromolecular networks that can maintain a large amount of an aqueous solvent within their structures. A new kind of amphiphilic cetylated polyethyleneimine was prepared, and then, polylactic-co-glycolic acid cetylated polyethyleneimine/HA NPs (PCPH NPs) were synthesized by self-assembly as a new type of gene-delivering system. The delivery ability and cytotoxicity of PCPH NPs were determined as plasmid DNA vectors to transfect (liver hepatocellular carcinoma in vitro) and displayed much lower cytotoxicity and higher gene-delivery efficiency than polyethyleneimine and commercial transfection reagents. ${ }^{64}$
Polyphenols (negative groups) of strawberry extracts linked with positively protonated amino groups of chitosan helped to achieve maximum encapsulation. This method can enhance the bioavailability and sustained release of phytochemicals having lower bioavailability. ${ }^{65}$ Curcumin and emu oil derived from emu birds (Dromaius novaehollandiae) have shown good results against inflammation. However, the delivery of curcumin reduce the health problem due to low solubility and poor transmission. Nanoemulsions were synthesized using emu oil, Cremophor, and Labrafil as the oil phase, surfactant, and cosurfactant. The antiinflammatory arthritic scoring, paw volume, biochemical, molecular, radiological, and histological tests showed important improvements in anti-inflammatory activity with formulations containing curcumin in combination with emu oil compared to pure curcumin. ${ }^{66}$ The environment friendly synthesis of biodegradable semiinterpenetrating hydrogel networks using $N, N^{\prime}$-methylenebis(acrylamide) (MBA) and carboxymethylcellulose has been performed. Then, AgNPs supported on hydrogel networks as nanoreactors have used $\mathrm{AgNO}_{3}$ and Azadirachta indica (Neem) plant extracts under ambient conditions by green chemistry methods. In addition, it was shown that the dispersion between polymeric nanocomposites and drug affects the degree of matrix swelling, its porosity, and diffusion release process. ${ }^{67}$

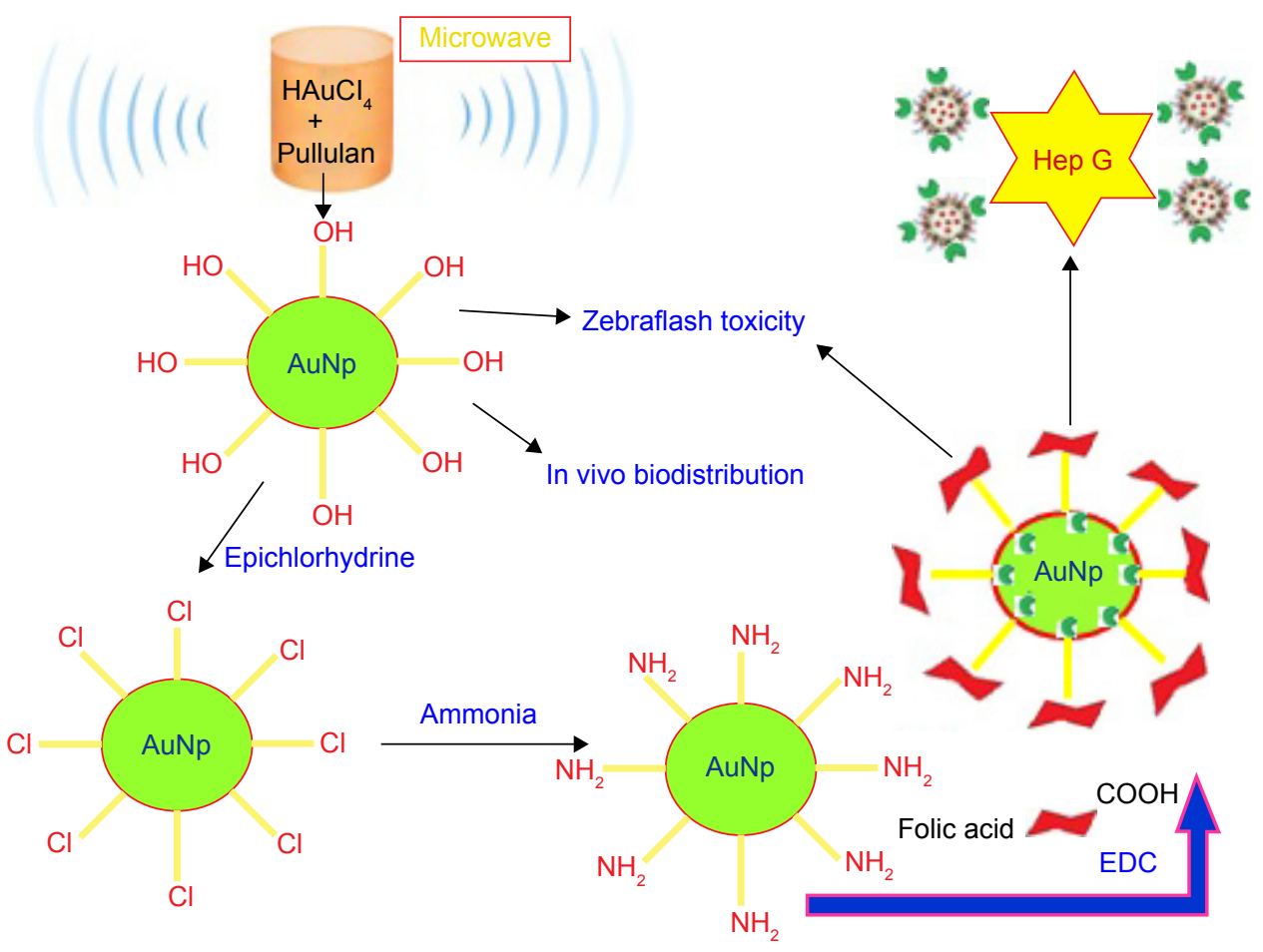

Figure $\mathbf{5}$ Schematic diagram for the synthesis of pullulan-stabilized AuNPs.

Abbreviations: AuNPs, gold nanoparticles; EDC, I-(3-Dimethylaminopropyl)-3-ethylcarbodiimide hydrochloride. 
Another fast, inexpensive, and renewable strategy for the preparation of AgNP has been performed in aqueous media using light emitted as the catalyst. This procedure is independent of NP seeds or harmful reactants. The prepared nanocomposite exhibited high antimicrobial activity against the tested bacterial strains. ${ }^{68}$ Other plants for the green synthesis of AgNPs as antimicrobials are Solanum tricobatum, Syzygium cumini, Centella asiatica, and Citrus sinensis extracts. These safe green chemistry AgNPs showed enhanced bactericidal activity against multiple drug-resistant human pathogens. The research presented showed the potential role of in vitro derived plantlet extracts for the practical synthesis of antimicrobial AgNPs, which can be applied as nanodrugs in many treatments. ${ }^{69}$ For the control of microbial infections, another cheap and green strategy for the preparation of AgNPs uses the cell-free supernatant from Delftia species with results demonstrating that the prepared NPs can be used as both antifungal agents and drug delivery agents. ${ }^{70}$

Anjum and Abbasi reported the application of a valuable endangered pharmaceutical plant species, Phlomis bracteosa, in the preparation of AgNPs. These biosynthesized AgNPs showed more enhanced bactericidal activity compared to multiple drug-resistant human pathogens. ${ }^{71}$ An interesting report in this area was recently published based on the very fast photosynthesis of AgNPs based on an extract from the aerial parts of Artemisia marschalliana Sprengel. The photosynthesized AgNPs displayed exceptional antibacterial activities compared to the assayed pathogenic bacteria, such as $S$. aureus, Bacillus cereus, Acinetobacter baumannii, and P. aeruginosa. ${ }^{72}$ Green-synthesized AgNPs from longan peel powder also demonstrated an antitumor ability of AgNPs, proposing that they have a good capability in lung cancer chemoprevention and chemotherapy, especially for earlystage intervention. ${ }^{73}$

Ginsenosides or panaxosides are a big group of natural steroid glycosides and triterpene saponins. Materials in this class exist nearly in the plant genus Panax (ginseng), which has a historical application in traditional medicine, especially in pharmacological compounds. Green ginsenoside NPs have important roles for carrying various insoluble drugs. Dai et $\mathrm{al}^{74}$ reported stable NPs from ginsenoside and anticancer drugs, with enhanced antitumor effects in vitro and in vivo compared to free drugs. Research by Vimala et $\mathrm{al}^{75}$ has indicated the potential of zinc oxide $(\mathrm{ZnO}) \mathrm{NPs}$ as a carrier for DOX, which focuses on the significance of progressing novel green chemistry-based strategies for introducing safe sources of NPs.

\section{CNTs}

CNTs, with individual physical and chemical properties, have become famous materials in cancer diagnosis and therapy. These compounds have most promising roles, for example, for detecting cancerous cells and carrying drugs or small therapeutic molecules to tumor cells. However, CNTs not only exhibit a high ability for drug loading but also exhibit a potential for cell penetration. Besides many applications of CNTs in drug delivery systems, some difficulty appears with low solubility, clumping or agglomeration, and long half-lives. Modification of single-walled nanotubes (SWNTs) with different compounds has changed solubility and tumor targeting/drug delivery. Such functionalization has reduced their cytotoxicity and altered the function of immune cells, such as PEG and polyethylenimine (PEI), ${ }^{76}$ cationic glycopolymers, ${ }^{77}$ vinblastine, ${ }^{78}$ and Lentinan. ${ }^{79}$

Huang et al reported good results using mice-bearing syngeneic murine squamous cell tumors. They intratemporally injected single-walled CNTs (SWCNTs) and irradiated the cancer cells with a low power NIR laser for 10 minutes. They exhibited a thermal ablation threshold at a maximum tumor temperature of $\sim 55^{\circ} \mathrm{C}$, and a reduction in cancer cell growth was observed. ${ }^{80}$ In the same research, Liu et al ${ }^{81}$ systematically studied the dependency between the length and density of PEGylation on the SWCNT surface and the biodistribution, tumor accumulation, and photothermal ablation ability of PEG-functionalized SWCNTs syringed intravenously into mice. Results showed long blood circulation half-lives for heavily PEGylated SWCNTs and high tumor uptake. This research proposed that the surface properties of CNTs are important to increase their unknown tumor-targeting abilities. ${ }^{81}$

Beside these applications, functional groups with oxygen on oxidized SWCNTs have been applied to link FA molecules with the SWNTs and solubility of the FAfunctionalized SWNTs can be easily increased in water and phosphate-buffered saline after a long time. These FA-functionalized SWNTs have great potential for applications as biocompatible SWNTs. ${ }^{82}$ An eco-friendly and safe graphene nanosheet-CNT-iron oxide $\left(\mathrm{GN}-\mathrm{CNT}-\mathrm{Fe}_{3} \mathrm{O}_{4}\right)$ NP hybrid has been used as a new composite for attaching and carrying antitumor drugs. ${ }^{83}$ Inadequate vascularization in biological meshes, resulting in limited host tissue incorporation, is related to abdominal wall defect repair after implantation. The sufficient release of exogenous angiogenic factors from biocompatible NPs could be a method to tackle this problem. Liu et al synthesized functionalized multiwalled CNTs (MWCNTs) by plasma polymerization to deliver vascular 
endothelial growth factor ${ }_{165}\left(\mathrm{VEGF}_{165}\right)$. The new $\mathrm{VEGF}_{165}{ }^{-}$ controlled released compound was inserted into porcine small intestinal submucosa (PSIS) to build a composite scaffold. Scaffolds incorporating $\mathrm{VEGF}_{165}$-supported functionalized multiwalled nanotubes (MWNTs) were implanted into rats to improve abdominal wall defects. The synthesis and application of functionalized MWNT-delivering VEGF $_{165}$ caused an increase in tissue repair rate by ensuring a maintained growth factor release. ${ }^{84}$ Betulinic acid (BA), with low solubility in water, supported on oxidized multiwalled CNTs (MWCNT-COOH) for increased delivery ability into tumor cells with high safety, has been prepared. The attachment of BA to the MWCNT-COOH nanodelivery vehicle was determined to be $\sim 14.5 \%-14.8 \%(\mathrm{w} / \mathrm{w})$. The release profiles exhibited that $\sim 98 \%$ of BA could be released. The biocompatibility study showed that MWCNT-BA at concentrations $<50 \mu \mathrm{g} / \mathrm{mL}$ revealed high safety effects for mouse embryo fibroblast cells after 72 hours of treatment. The antitumor activity of MWCNT-BA seemed to be more sensitive to a human lung cancer cell line than to a human liver cancer cell line, with the half maximal inhibitory concentration values of 2.7 and $11.0 \mu \mathrm{g} / \mathrm{mL}$, respectively. Their results form a fundamental area for more research concerning the impact of different MWCNT-BA formulations against cancer cells. ${ }^{85}$

The photothermal effect of SWCNTs modified with the antitumor drug DOX for targeting and accelerated destroying of breast cancer cells has been studied by Jeyamohan et al. A targeted drug-delivery vehicle was prepared for selective destruction of breast cancer cells with PEG-biofunctionalized and DOX-supported SWCNTs linked with FA. In their work, in vitro drug delivery studies showed that the drug (DOX) bound at physiological $\mathrm{pH}$ values ( $\mathrm{pH} 7.4$ ) and was released only at a lower $\mathrm{pH}$, ie, lysosomal $\mathrm{pH}$ values ( $\mathrm{pH} 4.0$ ), which is the characteristic $\mathrm{pH}$ of a tumor. SWCNTs have good optical absorbance. The optical properties of SWCNTs produce a strategy for the selective photothermal ablation in cancer treatment. Results of in vitro tests indicated that a laser was useful in preventing the growth of cancer cells, while protecting normal cells. When the laser effect was combined with DOX-conjugated SWCNTs, a sustained and accelerated killing of breast cancer cells was found. Thus, this nanodrug-delivery compound, made of a laser, drug, and SWCNTs, can be a valuable selective modality with good treatment potential and high safety for cancer therapy. ${ }^{86}$ A short description of the synthesis and design of SWCNTs as breast cancer drug delivery is shown in Figure 6.

Intracellular delivery ability is an important factor affected by using CNTs in cancer treatment. For this purpose, a small molecule chitosan linked with a transactivator of transcription (TAT) peptide was added to the noncovalent modification of MWCNTs, for a highly efficient drug delivery system for tumor therapy. The TAT-chitosan-conjugated MWCNTs
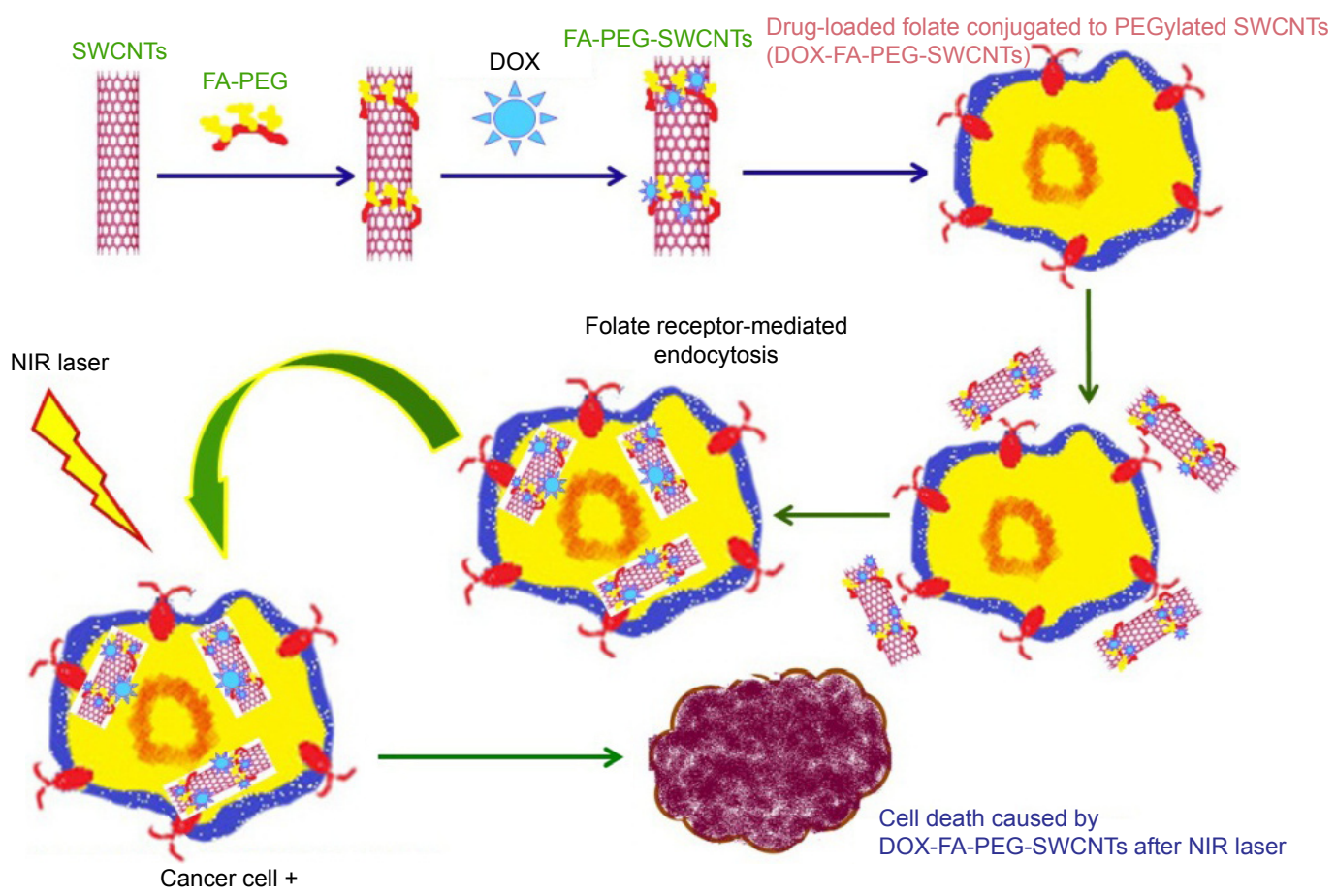

Figure 6 Schematic image of the modification of SWCNTs with the antitumor drug DOX for targeting and accelerated destroying of breast cancer. Abbreviations: DOX, doxorubicin; FA, folic acid; PEG, poly(ethylene glycol); SWCNTs, single-walled carbon nanotubes; NIR, near-infrared. 
(MWCNTs-TC) have significant promising properties, such as water solubility, low toxicity, cell-penetrating capability, and accumulation in tumors. Experiments show that MWCNTs-TC were essentially safe with high water solubility, and they were more efficient in terms of cancer-targeted intracellular transport both in vitro and in vivo as compared with chitosan-modified MWCNTs (MWCNTs-CS), proposing a new and good power for the use of MWCNTs-TC in cancer treatment. ${ }^{87}$

The high aspect ratio of CNTs allows for the attachment of compounds that enhance the functionality of the drug vehicle. Considering this, the use of CNTs as a multifunctional insulin carrier may be an interesting prospect to explore. The carboxylic acid groups were functionalized on the sidewalls of SWCNTs followed by diimidation to form amide bonds with the amine groups of the insulin. The incorporation of insulin further increased the solubility of SWCNTs in a biological solution for the tested period of 5 months. A bicinchoninic acid assay confirmed that $0.42 \mathrm{mg}$ of insulin could be attached to every $1 \mathrm{mg}$ of carboxylated SWCNTs. With the successful conjugation of insulin to SWCNTs, it opens up the potential use of SWCNTs as an insulin carrier, which is in need of further biological studies. ${ }^{88}$
Figure 7 shows the mechanism of the attachment of carboxylic acid groups on the surface of SWCNTs.

\section{Biodrug delivery vehicles}

Biotechnological studies today have mainly focused on safe and efficient nanodelivery based on protein and lipid compounds. Some have been developed with folate-decorated cationic liposomes for malignant melanoma treatment as pointed out by Chen et al, who described an economic preparation of hypoxia-inducible factor 1-alpha low interposing RNA-attached folate-modified cationic liposomes by a film hydration method. The antimelanoma activity substantially reduced the production of hypoxia-inducible factor 1-alphaassociated protein and interfered with the apoptosis of hypoxia-tolerant melanoma cells. ${ }^{89}$

Cancer nanotherapeutics is beginning to overwhelm global research and is viewed to be a revolutionary treatment regime in the medical field. This investigation describes the development of a stable nanostructured lipid carrier (NLC) system as a carrier for tamoxifen (TAM). Besides, the equivalent cytotoxicity of TAM and TAM-NLC to human (MCF-7) and mice (4T1) mammary breast cancer cell lines was observed. Incubating the formulation at a physiological

\section{Carboxylated CNTs}
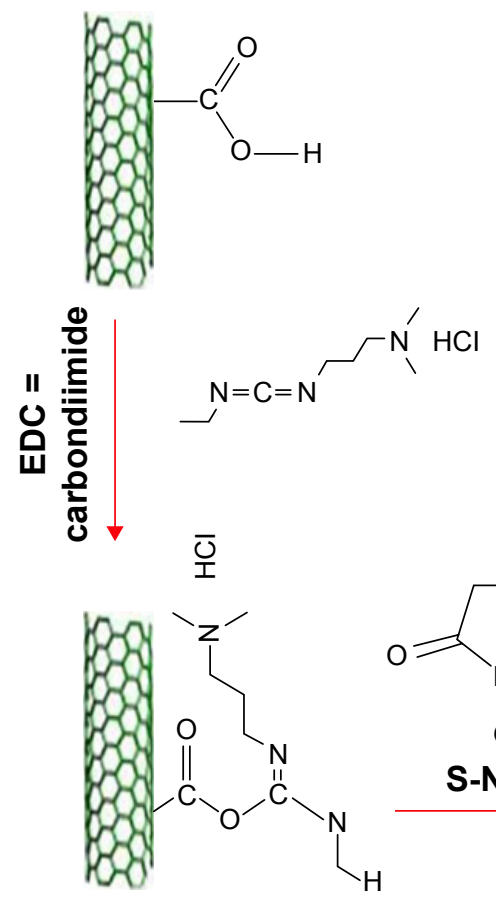

Unstable amine-reactive
O-acylisourea ester

\section{Amide bond with insulin}
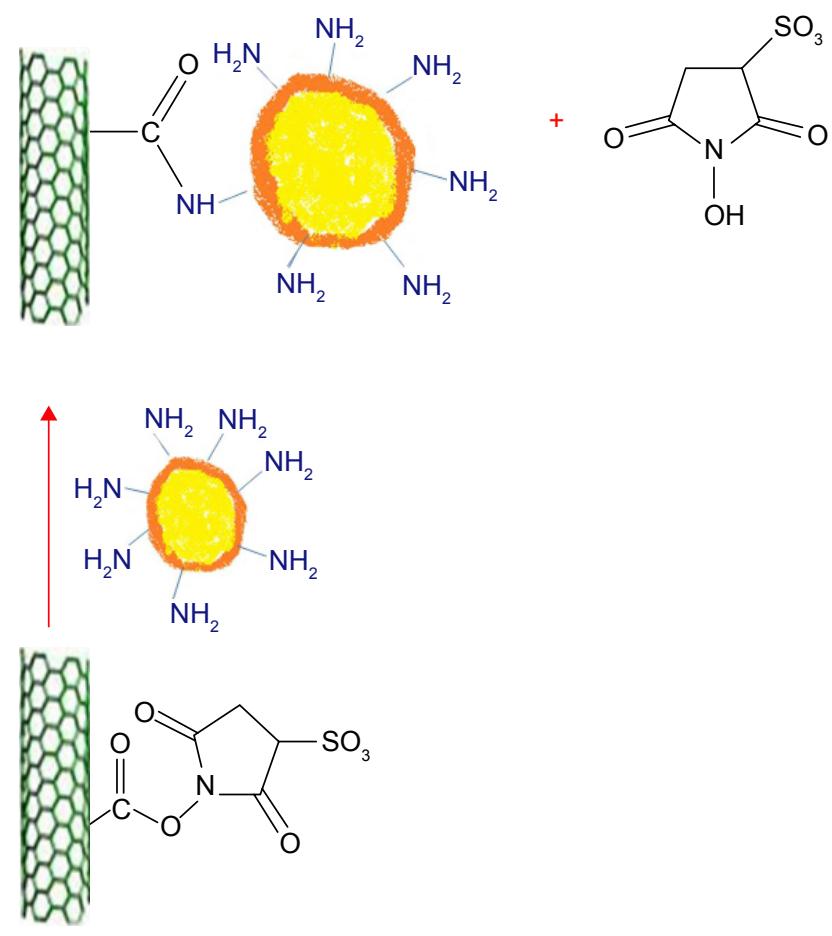

\section{Semistable amine-reactive NHS ester}

Figure 7 One example of using CNTs as a multifunctional insulin carrier.

Abbreviations: CNTs, carbon nanotubes; EDC, I-(3-Dimethylaminopropyl)-3-ethylcarbodiimide hydrochloride; NHS, N-hydroxysulfosuccinimide. 
$\mathrm{pH}$ resulted in a reduced Ostwald ripening rate but without any significant change in the absorptivity. The results of the study also suggested that TAM-NLC is a promising drug delivery system for breast cancer therapy. This is the first encouraging report on the in vitro effect of TAM-NLC against human and mouse mammary adenocarcinoma cell lines..$^{90}$

The high loading abilities and enhanced release for heparinbased nanocomplexes supported with placental growth factor (PlGF) and for those loaded with both PlGF and bone morphogenic protein-2 (BMP-2) are preparing the way for increased favorable bone cell functions while maximizing toxicity. This new nanocomplex shows good potential in the bone regeneration process. ${ }^{91}$ An outline for preparing the heparin-based nanocomplexes supported with PlGF is shown in Figure 8.

A DNA vaccine, namely plasmid internal ribosome, was used against Newcastle disease. Vaccination was performed using the DNA vaccine alone or as a mixture of the DNA and dextran-spermine (D-SPM), a NP applied for DNA delivery. Furthermore, the determination of different virus titers and contact routes for discussion to test the ability of different immunizations will help progress NP DNA vaccines. ${ }^{92}$
Novel colloidal nanocarriers can be used as effective and nontoxic drug delivery vehicles for naftifine. Naftifine-loaded microemulsions of colloidal nanocarriers have been constructed for dermal delivery. The in vitro pig skin and in vivo human penetration experiments showed the effect of microemulsions in the increased power of naftifine delivery. ${ }^{93}$

Lipid nanotubes (LNTs) are some of the most helpful materials for drug delivery and targeting. LNTs made of special compounds called AQUA (AQ-NH- $\left(\mathrm{CH}_{2}\right)_{10} \mathrm{COOH}$ ) (AQ: anthraquinone group) have been applied for carrying DOX. This novel lipid nanomaterial is safe, effective, biodegradable, biocompatible, and stable and is an enhanced compound for carrying drugs, which may be applied for colonic performance of DOX for colorectal cancer therapy. ${ }^{94}$ Four kinds of pluronics with varying hydrophilic-lipophilic balance (HLB) values were modified and conjugated on a polyamidoamine dendrimer. The dendrimer nanocarrier was conjugated with a highly lipophilic pluronic agent, exhibiting a higher drug-loading efficiency in comparison with other modified pluronics. The drug-loaded nanocarriers also indicated a high antiproliferative activity against breast cancer cells. ${ }^{95}$

Recently, a stabilized clindamycin-encapsulated polylactic acid with more drug delivery at the molecular level has been synthesized. These NPs exhibited high antimicrobial
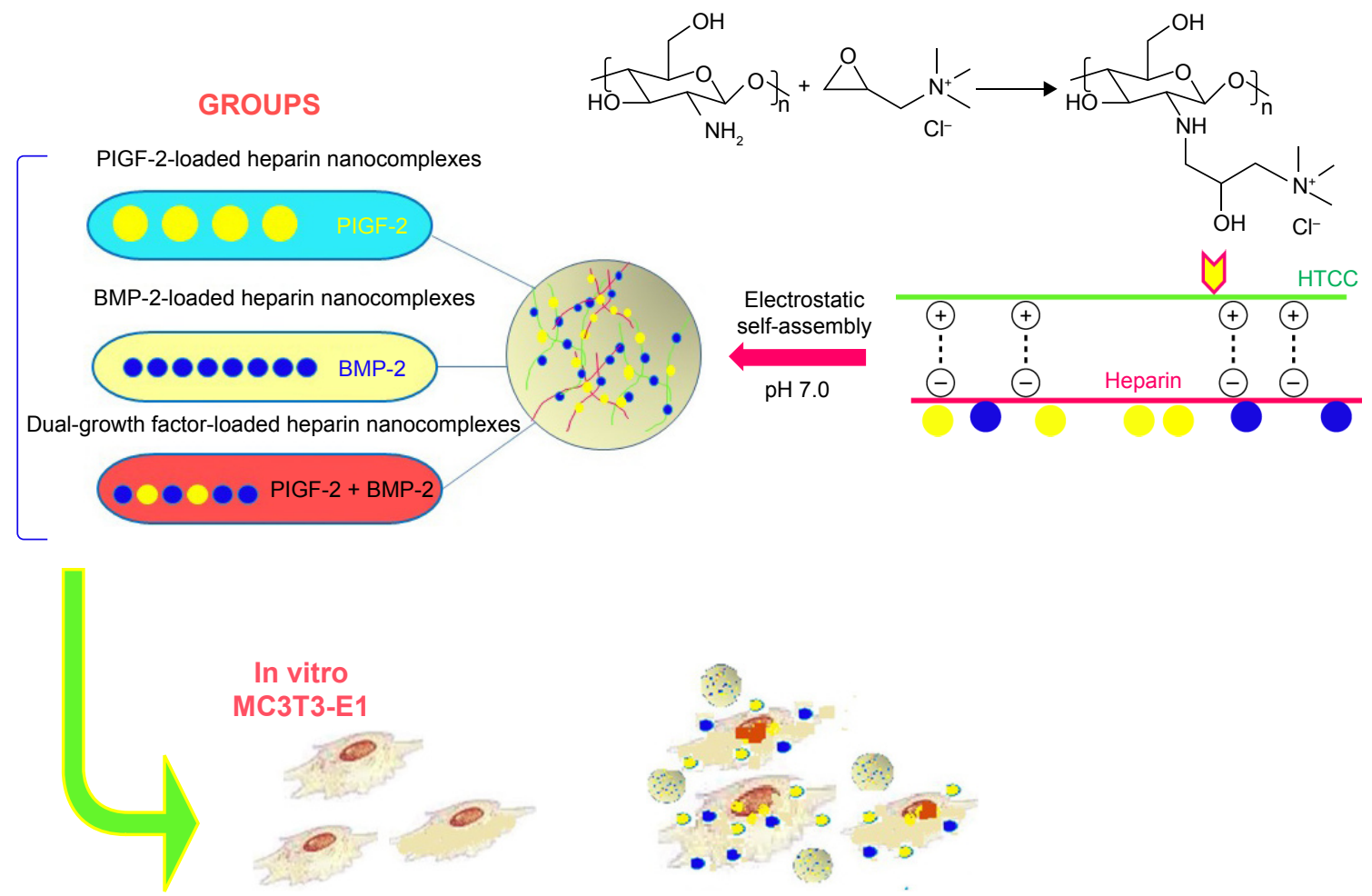

Osteogenic differentiation

Figure 8 Schematic illustration of the preparing and in vitro osteogenic effect of PIGF-2-/BMP-2-loaded heparin-HTCC nanocomplexes. Abbreviations: BMP-2, bone morphogenic protein-2; PIGF, placental growth factor; GF, Dual-growth factor-loaded; HTCC, heparin-N-(2-hydroxyl) propyl-3-trimethyl ammonium chitosan chloride. 
activity against two pathogenic bacteria Streptococcus faecalis and B. cereus. Also, the obtained experimental data proposed that the encapsulation of clindamycin with polylactic acid significantly increased the delivery of the drug. Phenanthriplatin, cis-[Pt $\left(\mathrm{NH}_{3}\right)_{2} \mathrm{Cl}($ phenanthridine $\left.)\right]\left(\mathrm{NO}_{3}\right)$, is a cationic monofunctional DNA-binding platinum(II) antitumor drug system with enhanced potential and cellular response profile. This nanodrug delivery vehicle is one of the best choices to investigate the delivery of numerous cancer drugs. ${ }^{96}$

A biodegradable and safe "bioinspired disassemblyreassembly strategy" method was used to reconstruct lipoprotein NPs (RLNs) using dehydrated human lipids and native apolipoproteins. These safe RLNs were designed to display the structure and properties of natural lipoproteins for efficient drug carrying and increased anticancer drug ability. ${ }^{97}$ One of the most important kinds of biodrug delivery vehicles is the use of modified cell-penetrating peptides with other drug vectors, which increase stability during blood circulation, and also progress the new synthesis of targeted delivery systems. ${ }^{98}$ Over the past decade, nanocages have an important role in the progression and implementation of many useful tools for studies involving biology, green chemistry, and material sciences as well as nanodrug delivery. ${ }^{99-102}$ The advantages of nanocages for drug delivery are their ability to minimize premature drug degradation or interaction with its biological environment and to transport cargo to specific tissues. DOX-loaded ferritins are specifically internalized into cancer cells via interactions with overexpressed transferrin receptor 1. Natural structure-based nanocages (such as the ferritins, small heat shock proteins, and vaults) and most of the viruses (virus-like particles, cowpea chlorotic mottle virus, cowpea mosaic virus, bacteriophage, and drug loadings in protein-based nanocages) are all important examples. ${ }^{103}$

A safe and smart $\mathrm{pH}$-sensitive nanocompound drug delivery with high drug delivery ability was prepared by conjugating DOX to xyloglucan (XG) using acid-cleavable hydrazone bonds and, then, encapsulating DOX by the self-assembly of XG-DOX conjugates to increase drug delivery into the tumor cells. ${ }^{104}$ The toxicity effects, such as the risk of bleeding due to heparin's anticoagulant activity, decreased using a novel combination nanosystem of low molecular weight heparin and ursolic acid (UA), which is also an angiogenesis inhibitor for tumor treatment. This synthesis led to high efficacy of anticancer treatment achieved with a reduction of angiogenesis, which leads to tumor metastasis. ${ }^{105}$ In recent research, it was reported that synthetic phosphoethanolamine has a good ability for inducing cell death in different tumor cell lines without side effects. However, its safety and selectivity against tumor cells could be maximized during encapsulation in cationic liposomes, such as dioctadecyldimethylammonium chloride, via electrostatic interactions between liposomes and tumor cell membranes. ${ }^{106}$

Quercetin (Que) is a famous anticancer drug, with low water solubility. A lecithin-based mixed polymeric micelle (LMPM) delivery vehicle has been used to increase the solubility and bioavailability of Que. An in vivo pharmacokinetic experiment showed that Que-LMPMs had higher area under a concentration-time curve and a longer halflife, causing more bioavailability compared to a free Que injection. ${ }^{107}$ The PLGA-lovastatin-chitosan-tetracycline NP is a great drug delivery vehicle for tetracycline and lovastatin for the bone regeneration process. The bone regeneration capability of PLGA NPs in three-walled defects in beagle dogs was determined. The obtained data showed that PLGA-lovastatin-chitosan-tetracycline NPs are biocompatible and can serve as antibacterial agents to decrease implant infection. ${ }^{108}$

Using host-guest interactions, a kind of linearhyperbranched supramolecular amphiphile and its assembled system for drug encapsulation and DNA delivery were prepared. Amine-attached $\beta$-CD-centered hyperbranched polyglycerol and linear adamantane-terminated octadecane molecules were designed to spontaneously link together and then self-assemble into a nanoscale system. Based on hydrophilic agents, DOX $\cdot \mathrm{HCl}$ was loaded into the hollow cavity of the vesicles. The nanovesicles showed maximum serum-tolerant transgene potential and specifically higher safety compared to the gold gene delivery systems. This system is a suitable delivery system for cationic supramolecular vesicles for stimulus-responsive drug/DNA transport. ${ }^{109}$ Transformable core-shell-based nanocarriers are an attractive system, which can enzymatically assemble into microsized extracellular depots at the tumor site with the assistance of hyaluronidase, an overexpressed enzyme at the tumor micromedia. The experimental data showed that core-shell based nanogel (CS-NG) can sufficiently release combinational anticancer drugs (tumor necrosis factor [TNF]-related apoptosis containing ligand [TRAIL] and antiangiogenic cilengitide) toward the membrane of tumor cells and endothelial cells at the acidic cancer cell microenvironment, respectively. Considerable cytotoxicity on tumor cells and high antitumor capability were gained by applying CS-NG, which was related to their inhibition of cellular internalization and prolonged retention time in vivo. ${ }^{110}$ Figure 9 shows the action of transformable core-shell-based nanocarrier schematically. ${ }^{110}$ Examples of green bionanodrug delivery vehicles are listed in Table 4. 


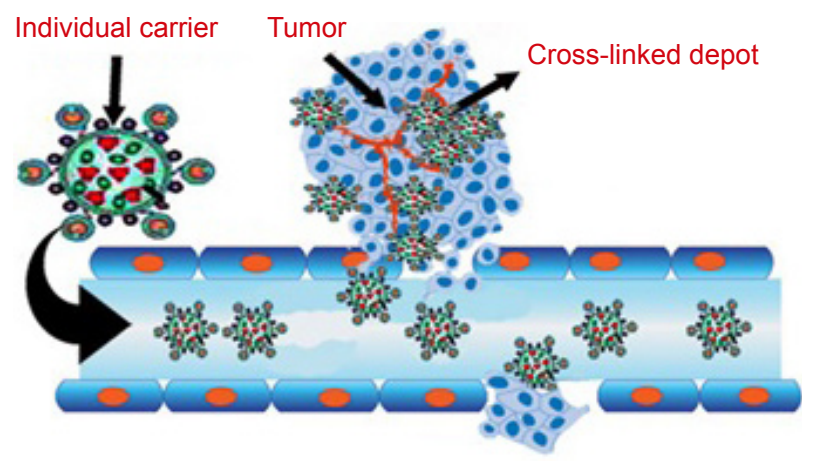

Figure 9 Schematic image of transformable core-shell-based nanocarriers.

Comparing the results listed in Tables 2 and 4 shows more drug delivery nanocomposites constructed based on polymer structures that have been used in cancer treatment or the transport of drugs to tumor cells. However, in between these nanocomposites, some nanocomposites can be seen to have a significant role in the treatment of other diseases, such as the delivery of drugs in a porcine coronary implant model, control of vancomycin (antibacterial) release, and the treatment of infrapopliteal lesions. Also, in the process of the synthesis of these nanocomposites, the final product has had a lower toxicity and side effects in agreement with the third rule of the 12 principles of green chemistry. In fact, general studies in the field of nanocomposites have shown that the most important goal in the synthesis of drugs is the achievement of healthier products. In comparison with nanocomposites, natural and biodegradable bionanodrug delivery vehicles have been made of lipids and protein structures. These particles are also used in the transfer of anticancer drugs. The less hazardous chemical syntheses, using safer solvents and auxiliaries, and the design for the degradation of the 12 principles of green chemistry in the synthesis of this group of drug carriers have been closely considered.

Finally, nanocolloids may be the breakout for an important change in using NPs. For example, anti-inflammatory and analgesic activities have been observed for novel aspirin oil-in-water $(\mathrm{O} / \mathrm{W})$ nanoemulsions and water-in-oil-in-water $(\mathrm{W} / \mathrm{O} / \mathrm{W})$ nanomultiple emulsions generated using ultrasound cavitation techniques. The anti-inflammatory activities of nanoemulsions and nanomultiple emulsions were determined using the $\lambda$-carrageenan-induced paw edema model. The analgesic activities of both nanoformulations were determined using acetic acid-induced writhing response and hot plate assays. For comparison, the effect of pretreatment with blank nanoemulsions and reference aspirin suspensions was also studied for their anti-inflammatory and antinociceptive activities. The results showed that oral administration of nanoemulsions and nanomultiple emulsions containing aspirin significantly reduced paw edema induced by $\lambda$-carrageenan injection. The experimental study suggests that nanoemulsions and nanomultiple emulsions produced pronounced anti-inflammatory and analgesic effects in rats and may be candidates as new nanocarriers for pharmacological non-steroidal anti-inflammatory drugs in the treatment of inflammatory disorders and alleviating pain. ${ }^{111}$

\section{Conclusion}

Nanosized polymers, low molecular weight molecules, and the biomacromolecules mentioned here are some of the

Table 4 Green bionanodrug delivery vehicles based on green chemistry principles

\begin{tabular}{llll}
\hline Bionanodrug delivery & Use & Green chemistry principles & References \\
\hline $\begin{array}{l}\text { Folate-decorated cationic liposomes } \\
\text { Heparin-based nanocomplexes }\end{array}$ & Malignant melanoma treatment & Safer solvents and auxiliaries \\
& $\begin{array}{l}\text { Release of placental growth factor-2 } \\
\text { and bone morphogenic protein-2 }\end{array}$ & Use of renewable feedstocks \\
Nanoparticle DNA vaccine & Newcastle disease & Design for degradation \\
Naftifine-loaded microemulsion & Cutaneous delivery & Designing safer chemicals \\
Lipid nanotubes & DOX delivery & Designing safer chemicals \\
$\begin{array}{l}\text { Pluronics with varing hydrophilic-lipophilic } \\
\text { conjuction }\end{array}$ & Breast cancer cell & Designing safer chemicals \\
$\begin{array}{l}\text { Stabilized clindamycin-encapsulated } \\
\text { poly lactic }\end{array}$ & Antimicrobial activity against & Safer solvents and auxiliaries \\
Lipoprotein nanoparticles & two pathogenic bacteria & & 92 \\
Cell-penetrating peptides as noninvasive & Drug delivery & Designing safer chemicals \\
transmembrane vectors & Drug delivery & Design for degradation \\
Conjugating DOX to xyloglucan & Drug delivered in the tumor region & Less hazardous chemical syntheses \\
A lecithin-based mixed polymeric micelle & Improve the solubility and & Inherently safer chemistry & 94 \\
& bioavailability of quercetin & for accident prevention & 96 \\
Amine-attached $\beta$-cyclodextrin & Drug/DNA transport & Less hazardous chemical syntheses & I09 \\
\hline
\end{tabular}

Abbreviation: DOX, doxorubicin. 
best candidates for the development of more efficient green chemistry methods for the synthesis of nanodrug delivery vehicles. Most of the developments with green processes have led to materials of low toxicity and high biocompatibility and have been designed by using plant extracts and biomaterials, such as proteins and lipids. As such, green chemistry methods have helped to avoid one of the biggest complications faced with today's NP drug delivery systems: toxicity. However, despite the research emphasized here, it is clear that much more work is needed to develop safe NPs for medicine, whether in the final product or the process used to make such effective NPs.

\section{Acknowledgment}

The authors would like to acknowledge Ilam University, Ilam, Iran, and Northeastern University, Boston, MA, USA, for their earnest cooperation in this research.

\section{Disclosure}

The authors report no conflicts of interest in this work.

\section{References}

1. Ravichandran S. Green chemistry- a potential tool for chemical synthesis. Int J Chem Tech Res. 2010;2(4):2188-2191.

2. White MA, Johnson JA, Koberstein JT, Turro NJ. Toward the syntheses of universal ligands for metal oxide surfaces: controlling surface functionality through click chemistry. J Am Chem Soc. 2006; 128(35):11356-11357.

3. Arruebo M, Fernandez-Pacheco R, Ibarra MR, Santamaria J. Magnetic nanoparticles for drug delivery. Nano Today. 2007;2(3):22-32.

4. Mornet S, Vasseur S, Grasset F, Duguet E. Magnetic nanoparticle design for medical diagnosis and therapy. J Mater Chem. 2004;14(21): 61-75.

5. Sun Z, Worden M, Thliveris JA, et al. Biodistribution of negatively charged iron oxide nanoparticles (IONPs) in mice and enhanced brain delivery using lysophosphatidic acid (LPA). Nanomedicine. 2016; 12(7):1775-1784.

6. Kumar CSSR, Mohammad F. Magnetic nanomaterials for hyperthermiabased therapy and controlled drug delivery. Adv Drug Deliv Rev. 2011; 63(9): 789-808.

7. Zhang Y, Sun C, Kohler N, Zhang M. Self-assembled coatings on individual monodispersed magnetite nanoparticles for efficient intracellular uptake. Biomed Microdevices. 2004;6(1):33-40.

8. Le VH, Rodriguez HL, Lee C, et al. A soft-magnet-based drug-delivery module for active locomotive intestinal capsule endoscopy using an electromagnetic actuation system. Sens Actuat A Phys. 2016;243:81-89.

9. Li J, Wu S, Wu C, et al. Versatile surface engineering of porous nanomaterials with bioinspired polyphenol coatings for targeted and controlled drug delivery. Nanoscale. 2016;8(16):8600-8606.

10. Liu J, Luo Z, Zhang J, et al. Hollow mesoporous silica nanoparticles facilitated drug delivery via cascade $\mathrm{pH}$ stimuli in tumor microenvironment for tumor therapy. Biomaterials. 2016;83:51-65.

11. Zhu X, Cao W, Chang B, et al. Polyacrylate/nanosilica causes pleural and pericardial effusion, and pulmonary fibrosis and granuloma in rats similar to those observed in exposed workers. Int J Nanomedicine. 2016; 11:1593-1605.

12. Song Y, Li X, Wang L, et al. Nanomaterials in humans: identification, characteristics, and potential damage. Toxicol Pathol. 2011;39(5): 841-849.
13. Pozdnyakov AS, Emelyanov AI, Kuznetsova NP, et al. Nontoxic hydrophilic polymeric nanocomposites containing silver nanoparticles with strong antimicrobial activity. Int $J$ Nanomedicine. 2016;11: 1295-1304.

14. Li X, Gong Y, Zhou X, et al. Facile synthesis of soybean phospholipidencapsulated MoS2 nanosheets for efficient in vitro and in vivo photothermal regression of breast tumor. Int $J$ Nanomedicine. 2016;11: 1819-1833.

15. Qian C, Chao W, Liang C, Weiwei H, Zhengping C, Zhuang L. Protein modified upconversion nanoparticles for imaging-guided combined photothermal and photodynamic therapy. Biomaterials. 2014;35(9):2915-2923.

16. Zhao MD, Cheng JL, Yan JJ, et al. Hyaluronic acid reagent functional chitosan-PEI conjugate with AQP2-siRNA suppressed endometriotic lesion formation. Int J Nanomedicine. 2016;11:1323-1336.

17. El-Meliegy E, Farag MM, Knowles JC. Dissolution and drug release profiles of phosphate glasses doped with high valency oxides. J Mater Sci Mater Med. 2016;27(6):108.

18. Das D, Patra P, Ghosh P, Rameshbabu AP, Dhara S, Pal S. Dextrin and poly(lactide)-based biocompatible and biodegradable nanogel for cancer targeted delivery of doxorubicin hydrochloride. Polym Chem. 2016;7(17):2965-2975.

19. Obayemi JD, Malatesta KA, Odusanya OS, et al. Abstract C60: injectable, biodegradable micro- and nano-particles loaded with prodigiosinbased drug for localized anticancer drug delivery. Cancer Epidemiol Biomarkers Prev. 2016;25(3):B40.

20. Li Q, Wen Y, Wen J, et al. A new biosafe reactive oxygen species (ROS)-responsive nanoplatform for drug delivery. RSC Adv. 2016;6: 38984-38989.

21. Sahiner N, Sagbas S, Aktas N. Preparation of macro-, micro-, and nanosized poly(Tannic acid) particles with controllable degradability and multiple biomedical uses. Polym Degrad Stab. 2016;129:96-105.

22. Bolu BS, Gecici EM, Sanyal R. Combretastatin A-4 conjugated antiangiogenic micellar drug delivery systems using dendron-polymer conjugates. Mol Pharm. 2016;13(5):1482-1490.

23. Tamai H, Igaki K, Kyo E, et al. Initial and 6-month results of biodegradable poly-L-lactic acid coronary stents in humans. Circulation. 2000; 102(4):399-404.

24. Kommareddy S, Amiji M. Poly(ethylene glycol)-modified thiolated gelatin nanoparticles for glutathione-responsive intracellular DNA delivery. Nanomedicine. 2007;3(1):32-42.

25. Carlyle WC, McClain JB, Tzafriri AR, et al. Enhanced drug delivery capabilities from stents coated with absorbable polymer and crystalline drug. J Control Release. 2012;162(3):561-567.

26. Peuster M, Wohlsein P, Brugmann M, et al. A novel approach to temporary stenting: degradable cardiovascular stents produced from corrodible metal-results 6-18 months after implantation into New Zealand white rabbits. Heart. 2001;86(5):563-569.

27. Peuster M, Hesse C, Schloo T, Fink C, Beerbaum P, von Schnakenburg C. Long-term biocompatibility of a corrodible peripheral iron stent in the porcine descending aorta. Biomaterials. 2006;27(28):4955-4962.

28. Peeters P, Bosiers M, Verbist J, Deloose K, Heublein B. Preliminary results after application of absorbable metal stents in patients with critical limb ischemia. J Endovasc Ther. 2005;12(1):1-5.

29. Hermawan H, Dube D, Mantovani D. Developments in metallic biodegradable stents. Acta Biomater. 2010;6(5):1693-1697.

30. Nanaki SG, Pantopoulos K, Bikiaris DN. Synthesis of biocompatible poly( $\varepsilon$-caprolactone)-block-poly(propylene adipate) copolymers appropriate for drug nanoencapsulation in the form of core-shell nanoparticles. Int J Nanomedicine. 2011;6:2981-2995.

31. Yousaf AM, Mustapha O, Kim DW, et al. Novel electrosprayed nanospherules for enhanced aqueous solubility and oral bioavailability of poorly water-soluble fenofibrate. Int J Nanomedicine. 2016;11:213-221.

32. Withey ABJ, Chen G, Nguyen TLU, Stenzel MH. Macromolecular cobalt carbonyl complexes encapsulated in a click-cross-linked micelle structure as a nanoparticle to deliver cobalt pharmaceuticals. Biomacromolecules. 2009;10(12):3215-3226. 
33. Tzeng SY, Yang PH, Grayson WL, Green JJ. Synthetic poly(ester amine) and poly(amido amine) nanoparticles for efficient DNA and siRNA delivery to human endothelial cells. Int J Nanomedicine. 2011; 2011(6):3309-3322.

34. Zhang Y, Zhou J, Yang C, et al. Folic acid-targeted disulfide-based cross-linking micelle for enhanced drug encapsulation stability and site-specific drug delivery against tumors. Int J Nanomedicine. 2016;11: 1119-1130.

35. Hao W, Liu D, Shang Y, Zhang J, Xu S, Liup H. pH-triggered copolymer micelles as drug nanocarriers for intracellular delivery. RSC Adv. 2016;6(35):29149-29158.

36. Van S, Das SK, Wang X, et al. Synthesis, characterization, and biological evaluation of poly(L- $\gamma$-glutamyl-glutamine)-paclitaxel nanoconjugate. Int J Nanomedicine. 2010;2010(5):825-837.

37. Mohammed M, Abdel-Gawad E, Awwad S, Kandil E, El-Agamy B. Therapeutic role of a synthesized calcium phosphate nanocomposite material on hepatocarcinogenesis in rats. Biochem Cell Biol. 2016;94(3): 279-288.

38. Tu H, Lu Y, Wu Y, et al. Fabrication of rectorite-contained nanoparticles for drug delivery with a green and one-step synthesis method. Int $J$ Pharm. 2015;493(1-2):426-433.

39. Lei Y, Hamada Y, Li J, et al. Targeted tumor delivery and controlled release of neuronal drugs with ferritin nanoparticles to regulate pancreatic cancer, progression. J Control Release. 2016;232(28):131-142.

40. Manuja A, Kumar B, Chopra M, et al. Cytotoxicity and genotoxicity of a trypanocidal drug quinapyramine sulfate loaded-sodium alginate nanoparticles in mammalian cells. Int J Biol Macromol. 2016;88: 146-155.

41. Mauro VD, Iafisco M, Salvarani N, et al. Bioinspired negatively charged calcium phosphate nanocarriers for cardiac delivery of MicroRNAs. Nanomedicine. 2016;11(8):891-906.

42. Slowing II, Trewyn BG, Giri S, Lin VSY. Mesoporous silica nanoparticles for drug delivery and biosensing applications. Adv Funct Mater. 2007;17(8):1225-1236.

43. Rodio M, Brescia R, Diaspro A, Intartaglia R. Direct surface modification of ligand-free silicon quantum dots prepared by femtosecond laser ablation in deionized water. $J$ Colloid Interface Sci. 2016;45: 242-248.

44. Yue S, Man W, Hongbing D, Yining W, Tao J. Beneficial effects of biomimetic nano-sized hydroxyapatite/antibiotic gentamicin enriched chitosan-glycerophosphate hydrogel on the performance of injectable polymethylmethacrylate. $R S C A d v$. 2015;5(110):91082-91092.

45. Zhu K, Ye T, Liu J, et al. Nanogels fabricated by lysozyme and sodium carboxymethyl cellulose for 5-fluorouracil controlled release. Int $J$ Pharm. 2013;441(1-2):721-727.

46. Gurunathan S, Han JW, Eppakayala V, Kim JH. Green synthesis of graphene and its cytotoxic effects in human breast cancer cells. Int $J$ Nanomedicine. 2013;8(1):1015-1027.

47. Renu GB, Kasturi M, Meifang Z, Muthupandian A, Nay MH, Sivakumar M. Sonochemical and sustainable synthesis of graphenegold ( $\mathrm{G}-\mathrm{Au})$ nanocomposites for enzymeless and selective electrochemical detection of nitric oxide. Biosen Bioelectron. 2017;87:622-629.

48. Renu GB, Kasturi M, Fiona NS, et al. The biogenic synthesis of reduced graphene oxide-silver (RGO-Ag) nanocomposite and its dual applications as antibacterial agent and cancer biomarker sensor. RSC Adv. 2016;6(43):36576-36587.

49. Ma N, Zhang B, Liu J, Zhang P, Li Z, Luan Y. Green fabricated reduced graphene oxide: evaluation of its application as nano-carrier for pH-sensitive drug delivery. Int J Pharm. 2015;496(2):984-992.

50. Zhu HY, Zhang SY, Ling Y, Meng G, Yang Y, Zhang W. pH-responsive hybrid quantum dots for targeting hypoxic tumor siRNA delivery. J Control Release. 2015;220:529-544.

51. Li Z, Xu W, Ang Y, et al. Quantum dots loaded nanogels for low cytotoxicity, $\mathrm{pH}$-sensitive fluorescence, cell imaging and drug delivery. Carbohydr Polym. 2015;121:477-485.

52. Bwatanglang IB, Mohammad F, Yusof NA, et al. Folic acid targeted $\mathrm{Mn}: \mathrm{ZnS}$ quantum dots for theranostic applications of cancer cell imaging and therapy. Int J Nanomedicine. 2016;11:413-428.
53. Feng T, Ai X, An G, Yang P, Zhao Y. Charge-convertible carbon dots for imaging-guided drug delivery with enhanced in vivo cancer therapeutic efficiency. ACS Nano. 2016;10(4):4410-4420.

54. Wang GH, Huang GL, Zhao Y, et al. ATP triggered drug release and DNA co-delivery systems based on ATP responsive aptamers and polyethylenimine complexes. J Mater Chem B. 2016;4(21):3832-3841.

55. Arshad A, Akram R, Iqbal S, et al. Aqueous synthesis of tunable fluorescent, semiconductor $\mathrm{CuInS}_{2}$ quantum dots for bioimaging. Arab $\mathrm{J}$ Chem. In press 2016.

56. Paciotti GF, Mayer L, Weinreich D, et al. Colloidal gold: a novel nanoparticle vector for tumour directed drug delivery. Drug Deliv. 2006; 11(3):169-183.

57. Sperling RA, Rivera Gil P, Zhang F, Zanella M, Parak WJ. Biological applications of gold nanoparticles. Chem Soc Rev. 2008;37(9):1896-1908.

58. Boisselier E, Astruc D. Gold nanoparticles in nanomedicine: preparations, imaging, diagnostics, therapies and toxicity. Chem Soc Rev. 2009; 38(9):1759-1782.

59. Kotla NG, Singh M, Maddiboyina B, Sunnapu O, Webster Thomas J. A novel dissolution media for testing drug release from a nanostructured polysaccharide-based colon specific drug delivery system: an approach to alternative colon media. Int J Nanomedicine. 2016;11:1089-1095.

60. Malathi S, Balakumaran MD, Kalaichelvan PT, Balasubramanian S. Green synthesis of gold nanoparticles for controlled delivery. Adv Mater Lett. 2013;4(12):933-940.

61. Lu R, Yang D, Cui D, Wang Z, Guo L. Egg white-mediated green synthesis of silver nanoparticles with excellent biocompatibility and enhanced radiation effects on cancer cells. Int J Nanomedicine. 2012;7:2101-2107.

62. Singh M, Kumar M, Manikandan S, Chandrasekaran N, Mukherjee A, Kumaraguru AK. Drug delivery system for controlled cancer therapy using physico-chemically stabilized bioconjugated gold nanoparticles synthesized from marine macroalgae Padina gymnospora. J Nanomed Nanotechol. 2014;S5:009.

63. Ganeshkumar M, Ponrasu T, Raja MD, Subamekala MK, Suguna L. Green synthesis of pullulan stabilized gold nanoparticles for cancer targeted drug delivery. Spectrochim Acta Mol Biomol Spectrosc. 2014; 130:64-67.

64. Zhu Y, Liang G, Sun B, Tian T, Hu F, Xiao Z. A novel type of selfassembled nanoparticles as targeted gene carriers: an application for plasmid DNA and antimicro RNA oligonucleotide delivery. Int $J$ Nanomedicine. 2016;11:399-411.

65. Pulicharla R, Marques C, Das RK, Rouissi T, Brar SK. Encapsulation and release studies of strawberry polyphenols in biodegradable chitosan nanoformulation. Int J Biol Macromol. 2016;88:171-178.

66. Jeengar MK, Rompicharla SVK, Shrivastava S, et al. Emu oil based nano-emulgel for topical delivery of curcumin. Int J Pharm. 2016; 506(1-2):222-236.

67. Gulsonbi M, Parthasarathy S, Bharat Raj K, Jaisankar V. Green synthesis, characterization and drug delivery applications of a novel silver/ carboxymethylcellulose - poly(acrylamide) hydrogel nanocomposite. Ecotoxicol Environ Saf. 2015;15:30145-30147.

68. Banasiuk R, Frackowiak JE, Krychowiak M, et al. Synthesis of antimicrobial silver nanoparticles through a photomediated reaction in an aqueous environment. Int J Nanomedicine. 2016;11:315-324.

69. Logeswari P, Silambarasan S, Abraham J. Ecofriendly synthesis of silver nanoparticles from commercially available plant powders and their antibacterial properties. Sci Iran. 2013;20(3):1049-1054.

70. Ganesh Kumar C, Poornachandra Y. Biodirected synthesis of Miconazole-conjugated bacterial silver nanoparticles and their application as antifungal agents and drug delivery vehicles. Colloids Surf B Biointerfaces. 2015;125:110-119.

71. Anjum S, Abbasi BH. Biomimetic synthesis of antimicrobial silver nanoparticles using in vitro-propagated plantlets of a medicinally important endangered species: Phlomis bracteosa. Int J Nanomedicine. 2016; 11:1663-1675.

72. Salehi S, Shandiz SAS, Ghanbar F, et al. Phytosynthesis of silver nanoparticles using Artemisia marschalliana Sprengel aerial part extract and assessment of their antioxidant, anticancer, and antibacterial properties. Int J Nanomedicine. 2016;11:1835-1846. 
73. He Y, Du Z, Ma S, et al. Effects of green-synthesized silver nanoparticles on lung cancer cells in vitro and grown as xenograft tumors in vivo. Int J Nanomedicine. 2016;11:1879-1887.

74. Dai L, Liu K, Si C, et al. Ginsenoside nanoparticle: a new green drug delivery system. J Mater Chem B. 2016;4(3):529-538.

75. Vimala K, Sundarraj S, Paulpandi M, Vengatesan S, Kannan S. Green synthesized doxorubicin loaded zinc oxide nanoparticles regulates the Bax and Bcl-2 expression in breast and colon carcinoma. Process Biochem. 2014;49(1):160-172.

76. Taghavi S, HashemNia A, Mosaffa F, Askarian S, Abnous K, Ramezani M. Preparation and evaluation of polyethylenimine-functionalized CNTs tagged with 5TR1 aptamer for targeted delivery of Bcl-xL shRNA into breast cancer cells. Colloids Surf B Biointerfaces. 2016;1(140):28-39.

77. Ahmed M, Jiang X, Deng Z, Narain R. Cationic glyco-functionalized single-walled CNTs as efficient gene delivery vehicles. Bioconjugate Chem. 2009;20(11):2017-2022.

78. Li Z, Tozer T, Alisaraie L. Molecular dynamics studies for optimization of noncovalent loading of vinblastine on single-walled CNT. J Phys Chem C. 2016;120(7):4061-4070.

79. Xing J, Liu Z, Huang Y, et al. Lentinan-modified CNTs as an antigen delivery system modulate immune response in vitro and in Vivo. ACS Appl Mater Interfaces. 2016;8(30):19276-19283.

80. Huang N, Wang H, Zhao J, Lui H, Korbelik M, Zeng H. Single-wall CNTs assisted photothermal cancer therapy: animal study with a murine model of squamous cell carcinoma. Laser Surg Med. 2010;42(9): 638-648.

81. Liu XW, Tao HQ, Yang K, Zhang SA, Lee ST, Liu ZA. Optimization of surface chemistry on single-walled CNTs for in vivo photothermal ablation of tumors. Biomaterials. 2011;32(1):144-151.

82. Saltan N, Kutlu HM, Hur D, Iscan A, Say R. Interaction of cancer cells with magnetic nanoparticles modified by methacrylamido-folic acid. Int J Nanomedicine. 2011;6:477-484.

83. Fan X, Jiao G, Gao L, Jin P, Li X. The preparation and drug delivery of a graphene-CNT- $\mathrm{Fe}_{3} \mathrm{O}_{4}$ nanoparticle hybrid. J Mater Chem. 2013; 1(20):2658-2664.

84. Liu Z, Feng X, Wang H, et al. CNTs as VEGF carriers to improve the early vascularization of porcine small intestinal submucosa in abdominal wall defect repair. Int J Nanomedicine. 2014;9:1275-1286.

85. Tan JM, Karthivashan G, Arulselvan P, Fakurazi S, Hussein MZ. Characterization and in vitro studies of the anticancer effect of oxidized CNTs functionalized with betulinic acid. Drug Des Dev Ther. 2014;8: 2333-2343.

86. Jeyamohan P, Hasumura T, Nagaoka Y, Yoshida Y, Maekawa T, Kumar DS. Accelerated killing of cancer cells using a multifunctional single-walled CNT-based system for targeted drug delivery in combination with photothermal therapy. Int J Nanomedicine. 2013; 8(1):2653-2667

87. Dong X, Liu L, Zhu D, Zhang H, Leng X. Transactivator of transcription (TAT) peptide-chitosan functionalized multiwalled CNTs as a potential drug delivery vehicle for cancer therapy. Int J Nanomedicine. 2015;3(10):3829-3840.

88. Ng CM, Loh HS, Muthoosamy K, Sridewi N, Manickam S. Conjugation of Insulin onto the sidewalls of single-walled carbon nanotubes (SWCNTs) through functionalization and diimide-activated amidation. Int J Nanomedicine. 2016;11:1607-1614.

89. Chen Z, Zhang T, Wu B, Zhang X. Insights into the therapeutic potential of hypoxia-inducible factor- $1 \alpha$ small interfering RNA in malignant melanoma delivered via folate-decorated cationic liposomes. Int $J$ Nanomedicine. 2016;11:991-1002.

90. How CW, Rasedee A, Manickam S, Rosli R. Tamoxifen-loaded nanostructured lipid carrier as drug delivery system: characterisation, stability assessment, and cytotoxicity. Colloids Surf B. 2013;112: 393-399.

91. Liu Y, Deng L-Z, Sun H-P, et al. Sustained dual release of placental growth factor-2 and bone morphogenic protein-2 from heparin-based nanocomplexes for direct osteogenesis. Int J Nanomedicine. 2016;11: 1147-1158.
92. Firouzamandi M, Moeini H, Hosseini SD, et al. Preparation, characterization, and in ovo vaccination of dextran-spermine nanoparticle DNA vaccine coexpressing the fusion and hemagglutinin genes against Newcastle disease. Int J Nanomedicine. 2016;11:259-267.

93. Erdal MS, Özhan G, Mat MC, Özsoy Y, Güngör S. Colloidal nanocarriers for the enhanced cutaneous delivery of naftifine: characterization studies and in vitro and in vivo evaluations. Int J Nanomedicine. 2016; 11:1027-1037.

94. Ilbasmis-Tamer S, Unsal H, Tugcu-Demiroz F, Kalaycioglu GD, Degim IT, Aydogan N. Stimuli-responsive lipid nanotubes in gel formulations for the delivery of doxorubicin. Colloid Surf B. 2016; 143:406-414

95. Nguyen TTC, Nguyen CK, Nguyen TH, Tran NQ. Highly lipophilic pluronics-conjugated polyamidoamine dendrimer nanocarriers as potential delivery system for hydrophobic drugs. Mater Sci Eng C. 2017;70:992-999.

96. Rauta PR, Das NM, Nayak D, Ashe S, Nayak N. Enhanced efficacy of clindamycin hydrochloride encapsulated in PLA/PLGA based nanoparticle system for oral delivery. IET Nanobiotechnol. 2016;10(4): 254-261.

97. Anna E, Czapar AE, Yao-Rong Zheng Y-R, et al. Tobacco mosaic virus delivery of phenanthriplatin for cancer therapy. ACS Nano. 2016;10(4):4119-4126.

98. Wang R, Gu X, Zhou J, et al. Green design "bioinspired disassemblyreassembly strategy" applied for improved tumor-targeted anticancer drug delivery. J Control Release. 2016;10(235):134-146.

99. Zhang D, Wang J, Xu D. Cell-penetrating peptides as noninvasive transmembrane vectors for the development of novel multifunctional drug-delivery systems. J Control Release. 2016;229:130-139.

100. Doll TA, Raman S, Dey R, Burkhard P. Nanoscale assemblies and their biomedical applications. J R Soc Interface. 2013;10(80):20120740.

101. Nune SK, Gunda P, Thallapally PK, Lin Y-Y, Laird Forrest M, Berkland CJ. Nanoparticles for biomedical imaging. Drug Deliv. 2009;6(11):1175-1194.

102. Whitesides GM. The 'right' size in nanobiotechnology. Nat Biotechnol. 2003;21:1161-1165.

103. Lee EJ, Lee NK, Kim I-S. Bioengineered protein-based nanocage for drug delivery. Adv Drug Deliv Rev. 2016;106(pt A):157-171.

104. Xie X, Luo S, Mukerabigwi JF, et al. Targeted nanoparticles from xyloglucan-doxorubicin conjugate loaded with doxorubicin against drug resistance. $R S C A d v$. 2016;6(31):26137-26146.

105. Li Y, Wu Y, Huang L, et al. Sigma receptor-mediated targeted delivery of anti-angiogenic multifunctional nanodrugs for combination tumor therapy. J Control Release. 2016;228:107-119.

106. Luna ACL, Saraiva GKV, Filho OMR, et al. Potential antitumor activity of novel DODAC/PHO-S liposomes. Int J Nanomedicine. 2016;11:1577-1591.

107. Chen LC, Chen YC, Su CY, Hong CS, Ho HO, Sheu MT. Development and characterization of self-assembling lecithin-based mixed polymeric micelles containing quercetin in cancer treatment and an in vivo pharmacokinetic study. Int J Nanomedicine. 2016;11:1557-1566.

108. Lee BS, Lee CC, Wang YP, et al. Controlled-release of tetracycline and lovastatin by poly(D,L-lactide-co-glycolide acid)-chitosan nanoparticles enhances periodontal regeneration in dogs. Int J Nanomedicine. 2016;11:285-297.

109. Yang B, Dong X, Lei Q, Zhuo R, Feng JX. Host-guest interactionbased self-engineering of nano-sized vesicles for co-delivery of genes and anticancer drugs. ACS Appl Mater Interfaces. 2015;7(39): 22084-22094.

110. Hu Q, Sun W, Lu Y, et al. Tumor microenvironment-mediated construction and deconstruction of extracellular drug-delivery depots. Nano Lett. 2016;16(2):1118-1126.

111. Tang SY, Sivakumar M, Ng AM, Shridharan P. Anti-inflammatory and analgesic activity of novel oral aspirin-loaded nanoemulsion and nano multiple emulsion formulations generated using ultrasound cavitation. Int J Pharm. 2012;430(1-2):299-306. 


\section{Publish your work in this journal}

The International Journal of Nanomedicine is an international, peerreviewed journal focusing on the application of nanotechnology in diagnostics, therapeutics, and drug delivery systems throughout the biomedical field. This journal is indexed on PubMed Central,

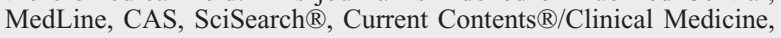

Journal Citation Reports/Science Edition, EMBase, Scopus and the Elsevier Bibliographic databases. The manuscript management system is completely online and includes a very quick and fair peer-review system, which is all easy to use. Visit http://www.dovepress.com/ testimonials.php to read real quotes from published authors.

Submit your manuscript here: http://www.dovepress.com/international-journal-of-nanomedicine-journal 